\title{
Non-linear Higgs portal to Dark Matter
}

\author{
I. Brivio, ${ }^{a}$ M.B. Gavela, ${ }^{a}$ L. Merlo, ${ }^{a}$ K. Mimasu, ${ }^{b}$ J.M. No, ${ }^{b}$ R. del Rey ${ }^{a}$ and V. Sanz ${ }^{b}$ \\ ${ }^{a}$ Departamento de Física Teórica and Instituto de Física Teórica, IFT-UAM/CSIC, \\ Universidad Autónoma de Madrid, \\ Cantoblanco, 28049, Madrid, Spain \\ ${ }^{b}$ Department of Physics and Astronomy, University of Sussex, \\ Brighton BN1 9QH, U.K. \\ E-mail: ilaria.brivio@uam.es, belen.gavela@uam.es, luca.merlo@uam.es, \\ k.mimasu@sussex.ac.uk, j.m.no@sussex.ac.uk, rocio.rey@uam.es, \\ v.sanz@sussex.ac.uk
}

Abstract: The Higgs portal to scalar Dark Matter is considered in the context of nonlinearly realised electroweak symmetry breaking. We determine the dominant interactions of gauge bosons and the physical Higgs particle $h$ to a scalar singlet Dark Matter candidate. Phenomenological consequences are also studied in detail, including the possibility of distinguishing this scenario from the standard Higgs portal in which the electroweak symmetry breaking is linearly realised. Two features of significant impact are: i) the connection between the electroweak scale $v$ and the Higgs particle departs from the $(v+h)$ functional dependence, as the Higgs field is not necessarily an exact electroweak doublet; ii) the presence of specific couplings that arise at different order in the non-linear and in the linear expansions. These facts deeply affect the Dark Matter relic abundance, as well as the expected signals in direct and indirect searches and collider phenomenology, where Dark Matter production rates are enhanced with respect to the standard portal.

Keywords: Beyond Standard Model, Effective field theories, Higgs Physics

ARXIV EPRINT: 1511.01099 


\section{Contents}

1 Introduction 1

2 The non-linear Higgs-portal 3

3 Dark Matter phenomenology $\quad 6$

3.1 Dark Matter relic density 8

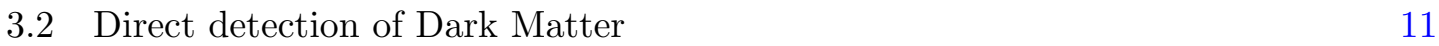

3.3 Invisible Higgs decay width 13

3.4 Dark Matter at the LHC: mono-X searches 14

$\begin{array}{lll}3.4 .1 & \text { Mono- } h \text { signatures } & 15\end{array}$

$\begin{array}{lll}3.4 .2 & \text { Mono-Z and mono-W searches } & 17\end{array}$

3.5 A comment on indirect detection of Dark Matter 20

4 Connection with the linear EFT expansion 21

5 Discussion and conclusions 23

$\begin{array}{ll}\text { A Feynman rules } & 26\end{array}$

B Contributions to the Dark Matter relic abundance $\quad 27$

$\begin{array}{lll}\text { C Impact of } \mathcal{A}_{1} \text { and } \mathcal{A}_{2} \text { for other choices of } c_{i} & 28\end{array}$

\section{Introduction}

The existence of Dark Matter (DM) cannot be explained within the Standard Model of particle physics (SM); its discovery and that of neutrino oscillations constitute the first clues of particle physics beyond the SM (BSM), whose nature awaits to be revealed. No interactions between the dark and the visible sectors have been observed ${ }^{1}$ although plausibly they may exist at some level [4]. These putative interactions must ensure the correct DM relic abundance as well as the stability of DM on cosmological timescales.

Three types of renormalisable (marginal or relevant, i.e. dimension $d \leq 4$ ) interactions between the SM fields and DM are possible: i) Higgs-scalar DM; ii) hypercharge field strength-vector DM; iii) Yukawa type couplings to fermionic DM. Being the lowest dimension couplings of the ordinary world to DM, they are excellent candidates - beyond gravitational interactions - to provide the first incursions into DM, i.e. to be the experimental "portals" into DM. In this paper we focus on the "Higgs portal" to real scalar DM.

\footnotetext{
${ }^{1}$ A claim for evidence of DM detection by the DAMA/LIBRA collaboration [1] has not been confirmed yet; also, some recent astrophysical analysis favouring visible-DM interactions [2] are open to alternative explanations [3].
} 
Assuming, as customary, a discrete $Z_{2}$ symmetry $[5,6]$ - under which the DM singlet scalar candidate $S$ is odd and the SM fields are even to ensure DM stability - the HiggsDM portal takes the form

$$
\lambda_{S} S^{2} \Phi^{\dagger} \Phi \longrightarrow \lambda_{S} S^{2}(v+h)^{2} \longrightarrow \lambda_{S} S^{2}\left(2 v h+h^{2}\right),
$$

where $\Phi$ denotes the $\mathrm{SU}(2)_{L}$ Higgs field doublet, $h$ the observed Higgs particle and $\lambda_{S}$ is the Higgs portal coupling; the right-hand side of the equation shows the DM-Higgs interaction in unitary gauge. The SM Higgs-DM portal in eq. (1.1) ("standard" portal all through this paper) has been extensively explored in the literature [7-25].

The nature of the Higgs particle itself also raises a quandary, though. The uncomfortable electroweak hierarchy problem - i.e. the surprising lightness of the Higgs particle - remains unsolved in the absence of any experimental signal in favour of supersymmetry or other palliative BSM solutions in which electroweak symmetry breaking (EWSB) is linearly realised. An alternative framework is that in which EWSB is non-linearly realised ("non-linear scenario" in short) and the lightness of the Higgs particle results from its being a pseudo-Goldstone boson of some global symmetry, spontaneously broken by strong dynamics at a high scale $\Lambda_{s}$. Much as the interactions of QCD pions are weighted down by the pion decay constant $f_{\pi}$, those of these new Goldstone bosons - including $h$ - will be weighted down by a constant $f$ such that $\Lambda_{s} \leq 4 \pi f[26]$, which may be distinct from the electroweak scale $v(v \ll f)$. Such an origin for a light Higgs particle was first proposed in the "composite Higgs" models in refs. [27-31], and has been interestingly revived in recent years in view of the fine-tunings of the hierarchy problem [32-35].

An interesting characteristic of the non-linear scenario is that the low-energy physical Higgs field may turn out not to be an exact electroweak doublet, and can be parametrised in the effective Lagrangian as a generic SM scalar singlet with arbitrary couplings [36-39]. In other words, the typical SM dependence on $(v+h)$ in eq. (1.1) is to be replaced by a generic polynomial $\mathcal{F}(h)$, implying the substitution of the standard portal in eq. (1.1) by the functional form

$$
\lambda_{S} S^{2}\left(2 v h+b h^{2}\right)
$$

where $b$ is an arbitrary, model dependent constant. The $h S S$ and $h h S S$ couplings - whose relative amplitude is fixed in the standard portal - are now decorrelated. This simple fact will be shown to have a deep impact on the estimates of the DM relic abundance, for which the relative strength of the DM coupling to one versus two $h$ particles plays a central role.

A further consequence of $h$ being treated as a generic scalar singlet is that its interactions are not necessarily correlated with those of the longitudinal components of the $W^{ \pm}$ and $Z$ gauge bosons, denoted by $\pi(x)$ in the customary $\mathbf{U}(x)$ matrix

$$
\mathbf{U}(x) \equiv e^{i \sigma_{a} \pi^{a}(x) / v} .
$$

While in linear BSM scenarios, $h$ and $\mathbf{U}(x)$ are components of the same object, i.e. the $\mathrm{SU}(2)_{L}$ Higgs doublet

$$
\Phi \equiv \frac{v+h}{\sqrt{2}} \mathbf{U}\left(\begin{array}{l}
0 \\
1
\end{array}\right)
$$


the independence of $h$ and $\mathbf{U}(x)$ in the non-linear Lagrangian induces a different pattern of dominant couplings. Although current measurements are compatible with the SM, present Higgs data allow for sizeable departures from $h$ being a pure Higgs doublet [40-42]. Indeed this characterisation is one of the most important quests of the LHC program, essential to unveil a putative non-linear origin of EWSB. A typical feature of the latter is the presence of relevant interactions that are expected to be further suppressed in the linear expansion [4350] (see also refs. [51, 52] for studies on the non-linear Higgs Lagrangian). It will be shown here that the bosonic couplings of $S$ also show this pattern, motivating the consideration of other interactions in addition to those in eq. (1.2) above. The ensemble will lead to potential smoking guns of the nature of the EWSB mechanism and of the Higgs particle. Distinct signals and (de)correlations in direct and collider DM searches will be discussed.

In summary, the focus of this paper is to explore the bosonic couplings of $S$ when EWSB is non-linearly realised. In particular, the effort will be directed to the comparison of the standard Higgs-portal encoded in eq. (1.1) and the equivalent interactions in the "non-linear Higgs portal". The paper is structured as follows: in section 2 the purely bosonic effective Lagrangian for the non-linear Higgs portal is introduced, discussing the differences between the non-linear setup and the standard Higgs portal. In section 3 the corresponding phenomenology is worked out, analysing the DM relic abundance, direct detection and bounds from colliders. In section 4 the impact of higher-dimension operators in the linear expansion is discussed and compared with the results for the non-linear portal. In section 5 we conclude.

\section{The non-linear Higgs-portal}

We restrict the analysis to the purely bosonic sector, except for the fermionic Yukawa-like terms. The relevant effective Lagrangian is derived below: it will be shown that only $v$ and the fermion and $S$ mass terms will remain as explicit scales.

This general Lagrangian may describe the leading effects of a plethora of models, for particular values of its coefficients. In those subjacent models, aside from fermion masses, several scales may be involved explicitly and implicitly, typically:

- The electroweak (EW) scale $v$, at which the effective Lagrangian is defined.

- The Goldstone-boson scale $f$ associated to the physical Higgs $h$, whose value does not need to coincide with $v$. Arbitrary functions $\mathcal{F}(h)$ would encode the Higgs dependence as a polynomial expansion in $h$.

- The scale $\Lambda_{s}$ of the high-energy strong dynamics, with $\Lambda_{s} \leq 4 \pi f$.

- The new physics scale $\Lambda_{\mathrm{DM}}$ characteristic of the DM interactions with the visible world, that is, the effective DM-Higgs portal scale, typically corresponding to the mass of a dark mediator.

- The mass of the scalar DM particle $m_{S}$. 
In the effective Lagrangian approach $v$ and the natural Goldstone boson scale $f$ are not separate parameters: $v$ is introduced as a fine-tuning requirement [53]. For instance it is customary to trade the $\mathcal{F}(h)$ polynomial dependence in powers of $h / f$ by an expansion in powers of $h / v$, with the arbitrary expansion coefficients absorbing the $v / f$ tuning. For the heavy scales, would $\Lambda_{\mathrm{DM}}$ coincide with $\Lambda_{s}$ or $f$, it would indicate a common origin for the Higgs and the DM candidate, as it occurs in models where both have their origin as Goldstone bosons of the high-energy strong dynamics [54-56]. Notice that, in such a scenario, the behavior of the $S$ field is expected to follow closely that of the Higgs particle: its dependence should be encoded in generic functions $\mathcal{F}(S)$ invariant under the $\mathbb{Z}_{2}$ symmetry (e.g. $\cos (S / f)$ ). The discussion will be kept here on a more general level and $\Lambda_{\mathrm{DM}}$ will be taken as an independent scale, although assuming $f \ll \Lambda_{\mathrm{DM}}$ in addition to plausibly $m_{S} \ll \Lambda_{\mathrm{DM}}$.

Furthermore, only the leading terms weighted down by $\Lambda_{\mathrm{DM}}$ and $\Lambda_{s}$ will be kept below, which in practice means no explicit dependence on them. Indeed, at leading order the expansion is tantamount to keeping the leading two-derivative terms of the electroweak chiral expansion [36, 57-60], supplemented by the $\mathcal{F}(h)$ dependences $[43-52,61,62]$ and the $S$ insertions: at this order the effective Lagrangian depends only on $v$, the fermion and $S$ mass terms, plus the operator coefficients.

The Lagrangian can be written as the sum of two pieces, with the second one encoding the DM interactions:

$$
\mathscr{L}=\mathscr{L}_{\mathrm{EW}}+\mathscr{L}_{S}
$$

with

$$
\begin{aligned}
\mathscr{L}_{\mathrm{EW}}= & -\frac{1}{4} W_{\mu \nu}^{a} W^{a \mu \nu} \mathcal{F}_{W}(h)-\frac{1}{4} B_{\mu \nu} B^{\mu \nu} \mathcal{F}_{B}(h)+\frac{1}{2} \partial_{\mu} h \partial^{\mu} h+ \\
& -\frac{v^{2}}{4} \operatorname{Tr}\left(\mathbf{V}_{\mu} \mathbf{V}^{\mu}\right) \mathcal{F}_{C}(h)+c_{T} \frac{v^{2}}{4} \operatorname{Tr}\left(\mathbf{T} \mathbf{V}_{\mu}\right) \operatorname{Tr}\left(\mathbf{T} \mathbf{V}^{\mu}\right) \mathcal{F}_{T}(h)-V(h)+ \\
& +i \bar{Q}_{L} \not D Q_{L}+i \bar{Q}_{R} \not D Q_{R}+i \bar{L}_{L} \not D L_{L}+i \bar{L}_{R} \not D L_{R}+ \\
& -\frac{v}{\sqrt{2}}\left(\bar{Q}_{L} \mathbf{U} \mathbf{Y}_{Q} Q_{R}+\text { h.c. }\right) \mathcal{F}_{Q}(h)-\frac{v}{\sqrt{2}}\left(\bar{L}_{L} \mathbf{U} \mathbf{Y}_{L} L_{R}+\text { h.c. }\right) \mathcal{F}_{L}(h)
\end{aligned}
$$

where

$$
\mathbf{D}_{\mu} \mathbf{U}(x) \equiv \partial_{\mu} \mathbf{U}(x)+i g \mathbf{W}_{\mu}(x) \mathbf{U}(x)-\frac{i g^{\prime}}{2} B_{\mu}(x) \mathbf{U}(x) \sigma_{3},
$$

with $\mathbf{W}_{\mu}(x) \equiv W_{\mu}^{a}(x) \sigma_{a} / 2$, and $W_{\mu}^{a}(x)$ and $B_{\mu}(x)$ denoting the SM gauge bosons. The scalar and vector chiral fields, $\mathbf{T}(x)$ and $\mathbf{V}(x)$, are defined as

$$
\mathbf{T}(x) \equiv \mathbf{U}(x) \sigma_{3} \mathbf{U}^{\dagger}(x), \quad \mathbf{V}_{\mu}(x) \equiv\left(\mathbf{D}_{\mu} \mathbf{U}(x)\right) \mathbf{U}^{\dagger}(x),
$$

with transformation properties under a (global) $\mathrm{SU}(2)_{L} \times \mathrm{SU}(2)_{R}$ symmetry given by:

$$
\mathbf{U}(x) \rightarrow L \mathbf{U}(x) R^{\dagger}, \quad \mathbf{T}(x) \rightarrow L \mathbf{T}(x) L^{\dagger}, \quad \mathbf{V}_{\mu}(x) \rightarrow L \mathbf{V}_{\mu}(x) L^{\dagger}
$$

After EWSB, $\mathrm{SU}(2)_{L} \times \mathrm{SU}(2)_{R}$ breaks down to the diagonal $\mathrm{SU}(2)_{C}$, which in turn is explicitly broken by the gauged hypercharge $\mathrm{U}(1)_{Y}$ and by the heterogeneity of the fermion masses. Equivalently, $\mathbf{T}(x)$ reduces to the Pauli $\sigma_{3}$ matrix, acting in this way as a spurion 
for the custodial symmetry. In eq. (2.2), the right-handed fermions have been gathered in $\mathrm{SU}(2)_{R}$ quark and lepton doublets, $Q_{R} \equiv\left\{u_{R}, d_{R}\right\}$ and $L_{R} \equiv\left\{\nu_{R}, e_{R}\right\}$, while the Yukawa couplings are encoded in $\mathbf{Y}_{Q} \equiv \operatorname{diag}\left\{Y_{U}, Y_{D}\right\}$ and $\mathbf{Y}_{L} \equiv \operatorname{diag}\left\{Y_{\nu}, Y_{\ell}\right\}$, i.e. it assumes Higgs couplings aligned with fermion masses. This Lagrangian is akin to the SM one written in chiral notation, but for the presence of the $\mathcal{F}(h)$ functions and the custodial breaking $c_{T}$ term, which is strongly constrained by data.

In eq. (2.1), the DM Lagrangian $\mathscr{L}_{S}$ at leading order in the $1 / \Lambda_{\mathrm{DM}}$ expansion reads

$$
\mathscr{L}_{S}=\frac{1}{2} \partial_{\mu} S \partial^{\mu} S-\frac{m_{S}^{2}}{2} S^{2} \mathcal{F}_{S_{1}}(h)-\lambda S^{4} \mathcal{F}_{S_{2}}(h)+\sum_{i=1}^{5} c_{i} \mathcal{A}_{i}(h),
$$

where the $\mathcal{A}_{i}$ operators form a basis:

$$
\left.\begin{array}{l}
\mathcal{A}_{1}=\operatorname{Tr}\left(\mathbf{V}_{\mu} \mathbf{V}^{\mu}\right) S^{2} \mathcal{F}_{1}(h) \\
\mathcal{A}_{2}=S^{2} \square \mathcal{F}_{2}(h) \\
\mathcal{A}_{3}=\operatorname{Tr}\left(\mathbf{T} \mathbf{V}_{\mu}\right) \operatorname{Tr}\left(\mathbf{T} \mathbf{V}^{\mu}\right) S^{2} \mathcal{F}_{3}(h) \\
\mathcal{A}_{4}=i \operatorname{Tr}\left(\mathbf{T V}_{\mu}\right)\left(\partial^{\mu} S^{2}\right) \mathcal{F}_{4}(h) \\
\mathcal{A}_{5}=i \operatorname{Tr}\left(\mathbf{T V}_{\mu}\right) S^{2} \partial^{\mu} \mathcal{F}_{5}(h)
\end{array}\right\} \quad \text { Custodial Violating }
$$

All $\mathcal{F}_{i}(h)$ functions in eqs. $(2.2),(2.6)$ and $(2.7)$ could be generically parametrised as an expansion in powers of $h$, e.g.

$$
\mathcal{F}_{i}(h) \equiv 1+2 a_{i} h / v+b_{i} h^{2} / v^{2}+\mathcal{O}\left(h^{3} / v^{3}\right) .
$$

Notice, however, that no $\mathcal{F}(h)$ functions accompany the Higgs, fermion and DM kinetic energies above, as they can be reabsorbed by field redefinitions without loss of generality [63]. Furthermore, in order to single out the impact of the DM couplings described by $\mathscr{L}_{S}$ and to ensure a clear comparison between the chiral and the linear setups, a simplification will be adopted in what follows for the $\mathcal{F}_{i}(h)$ functions in eq. (2.2):

$$
\mathcal{F}_{W}(h)=\mathcal{F}_{B}(h)=1, \quad \mathcal{F}_{C}(h)=(1+h / v)^{2}, \quad \mathcal{F}_{Q}(h)=\mathcal{F}_{L}(h)=(1+h / v),
$$

while due to the strong experimental constraints on $c_{T}$, we safely neglect its impact. Finally, it is useful to rewrite $\mathscr{L}_{S}$ as

$$
\mathscr{L}_{S}=\frac{1}{2} \partial_{\mu} S \partial^{\mu} S-\frac{m_{S}^{2}}{2} S^{2}-\lambda_{S} S^{2}\left(2 v h+b h^{2}\right)+\sum_{i=1}^{5} c_{i} \mathcal{A}_{i}(h)+\ldots
$$

by redefining the constant parameters in an obvious way, so that the $d \leq 4$ pure HiggsDM non-linear portal takes the form announced in eq. (1.2). The dots in eq. (2.10) stand for terms with more than two $h$ bosons and/or more than two $S$ fields, which are not phenomenologically relevant in the analysis below and are henceforth discarded. 
A pertinent question is how to complete the basis including fermionic couplings. There are two possible chiral fermionic structures to consider:

$$
\begin{array}{ll}
\bar{Q}_{L_{i}} \mathbf{U} Q_{R_{j}} S^{2} \mathcal{F}(h), & \bar{L}_{L_{i}} \mathbf{U} L_{R_{j}} S^{2} \mathcal{F}(h), \\
\bar{Q}_{L_{i}} \gamma_{\mu} Q_{L_{j}} \partial^{\mu} S^{2} \mathcal{F}(h), & \bar{L}_{L_{i}} \gamma_{\mu} L_{L_{j}} \partial^{\mu} S^{2} \mathcal{F}(h), \\
\bar{Q}_{R_{i}} \gamma_{\mu} Q_{R_{j}} \partial^{\mu} S^{2} \mathcal{F}(h), & \bar{L}_{R_{i}} \gamma_{\mu} L_{R_{j}} \partial^{\mu} S^{2} \mathcal{F}(h),
\end{array}
$$

where $i, j$ are flavour indices. The equations of motion, however, allow to relate a combination of the operators in eq. (2.11) to the operator $\mathcal{A}_{2}$, and a combination of the operators in eq. (2.12) to $\mathcal{A}_{4}$. In consequence, in order to avoid redundancies, a complete basis can be defined by the ensemble of all bosonic operators in eq. (2.7) plus those in eqs. (2.11) and (2.12), except for the two combinations of fermionic operators mentioned. Alternatively, the basis could be defined by all fermionic operators in eqs. (2.11) and (2.12) plus the bosonic ones in eq. (2.7), excluding $\mathcal{A}_{2}$ and $\mathcal{A}_{4}$. An optimal choice of the basis may depend on the data considered: in this paper the focus is set on the bosonic sector only, while the effects of introducing the fermionic one deserves a comprehensive future study, where flavour effects will also be taken into account [64]

In eq. (2.10), the $c_{i}$ 's $(i=1 \ldots 5)$ - together with the coefficients inside $\mathcal{F}_{i}(h)-$ parametrise the contributions of the $\mathcal{A}_{i}$ operators in the basis of eq. (2.7). These five effective operators describe interactions between two $S$ particles and: either two $W$ bosons, one or two $Z$ or $h$ bosons, or a $Z$ and a $h$ boson (see the Feynman rules in appendix A), inducing interesting phenomenological signatures as shown in the next section. $\mathcal{A}_{1}$ and $\mathcal{A}_{2}$ are custodial invariant couplings, in the sense that they do not contain sources of custodial symmetry breaking other than those present in the SM (hypercharge in this case). $\mathcal{A}_{3}$, $\mathcal{A}_{4}$ and $\mathcal{A}_{5}$ provide instead new sources of custodial symmetry violation. Nevertheless, the contribution of $\mathcal{A}_{4}$ to the $Z$ mass vanishes while that from $\mathcal{A}_{5}$ arises only at the two loop level (see appendix A), and no significant constraint on their operator coefficient follows the $\rho$ parameter and EW precision data; on the other hand, these observables do receive a one-loop contribution from $\mathcal{A}_{3}$. The bound on the corresponding coefficient is estimated to be around $c_{3} \sim 0.1$. Finally, notice that operators $\mathcal{A}_{1}, \mathcal{A}_{2}$ and $\mathcal{A}_{3}$ are CP-even, while $\mathcal{A}_{4}$ and $\mathcal{A}_{5}$ are CP-odd.

In summary, the non-linear portal in eq. (2.10) shows a much richer parameter space than the standard Higgs portal in eq. (1.1). The relationship between higher-dimension operators in the linear realisation of EWSB and the non-linear DM Higgs portal will be discussed in section 4 .

\section{Dark Matter phenomenology}

A wide variety of experimental data constrains the DM parameter space of Higgs portal scenarios described by the Lagrangian (2.10). The precise measurement of the DM density today, $\Omega_{\mathrm{DM}}$, performed by Planck [65] provides an upper bound on the relic abundance of $S$ particles, $\Omega_{S}$. Direct detection experiments set complementary limits on the strength of the DM-nucleon interactions, the current most stringent bounds coming from the Large 


\begin{tabular}{|lccccccc|}
\hline Observable & & \multicolumn{7}{c|}{ Parameters contributing } \\
& & $b$ & $c_{1}$ & $c_{2}$ & $c_{3}$ & $c_{4}$ & $c_{5}$ \\
\hline Thermal relic density & $\Omega_{S} h^{2}$ & $\checkmark$ & $\checkmark$ & $\checkmark$ & $\checkmark$ & $\checkmark$ & $\checkmark$ \\
DM-nucleon scattering in direct detection & $\sigma_{\mathrm{SI}}$ & - & - & $\checkmark$ & - & $\checkmark$ & - \\
Invisible Higgs width & $\Gamma_{\mathrm{inv}}$ & - & - & $\checkmark$ & - & - & - \\
Mono- $h$ production at LHC & $\sigma(p p \rightarrow h S S)$ & $\checkmark$ & - & $\checkmark$ & - & $\checkmark$ & $\checkmark$ \\
Mono- $Z$ production at LHC & $\sigma(p p \rightarrow Z S S)$ & - & $\checkmark$ & $\checkmark$ & $\checkmark$ & $\checkmark$ & $\checkmark$ \\
Mono- $W$ production at LHC & $\sigma\left(p p \rightarrow W^{+} S S\right)$ & - & $\checkmark$ & $\checkmark$ & - & $\checkmark$ & - \\
\hline
\end{tabular}

Table 1. Non-linear Higgs portal parameters affecting each of the observables considered. The standard Higgs-DM portal $b=1$ and all $c_{i}=0$.

Underground Xenon (LUX) experiment [66]. Upcoming experiments like XENON1T [67, 68] will further increase the sensitivity in DM direct detection. The couplings of DM to SM particles may be also probed at the LHC, with potential avenues including searches of invisible decay modes of the Higgs boson, and searches for mono- $X$ signatures, namely final states where one physical object $X$ is recoiling against missing transverse energy $\mathbb{E}_{T}$.

In the following we explore the rich phenomenology of non-linear Higgs portals. We first analyse the current constraints on the properties of DM coming from the DM relic abundance, direct detection limits from LUX and bounds on the invisible decay width of the Higgs boson. We then study the prospects for mono- $X$ signatures, with $X=h, W^{ \pm}$, $Z$, at the $13 \mathrm{TeV}$ run of the LHC. We also comment on the astrophysical signatures induced by the non-linear realisation, but defer a more detailed study of indirect detection in these models to future work. While our phenomenological study does not intend to exhaustively explore the parameter space of non-linear Higgs portals to DM, we do showcase all salient features of these scenarios and confront them with the standard Higgs portal. A list of the observables affected by each of the new terms in the DM Lagrangian ${ }^{2}(2.10)$ is shown in table 1 .

The non-linear DM-Higgs portal from eq. (2.10) is implemented in FeynRules [69] and interfaced to MicrOMEGAs [70] and MadGraph5_aMC@NLO [71] to compute the relevant observables. For the analysis of mono- $X$ signatures at the LHC, we use in addition the 1-loop FeynRules/NLOCT implementation of gluon-initiated mono- $X$ signatures via an s-channel mediator from [72], in order to capture the full momentum dependence in the production of mono- $X$ signatures via gluon fusion. In all cases, the standard portal corresponds to the choice $b=1, c_{i}=0$, and we compare it with different non-linear portal setups in which one of the parameters of the set $\left\{b, c_{i}\right\}$ is varied at a time. This approach ensures a clear and conservative phenomenological comparison between the standard and the non-linear portal scenarios, allowing to single out the physical impact of each effective operator.

\footnotetext{
${ }^{2}$ Our analysis has some overlap with the singlet scalar case of [56], which focuses on DM candidates that arise as pseudo-Goldstone bosons in specific composite Higgs models. While it is possible to identify a correspondence between our description and theirs for the case of $\mathcal{A}_{1}$ and $\mathcal{A}_{2}: \lambda_{S} \rightarrow \bar{\lambda}, c_{1} \rightarrow d_{4}(v / f)^{2}$, $c_{2} \rightarrow a_{d_{1}}(v / f)^{2}$, in the basis of [56] there is no equivalent of the operators $\mathcal{A}_{3}, \mathcal{A}_{4}, \mathcal{A}_{5}$. Moreover, the $(v / f)^{2}$ suppression in the analysis of [56] (where $f=800 \mathrm{GeV}, f=2.5 \mathrm{TeV}$ are considered) leads to a scan over values $\left|a_{d_{1}}\right| \times(v / f)^{2}<0.1,\left|d_{4}\right| \times(v / f)^{2}<0.1$, corresponding to a small subset of the parameter space probed in this work.
} 
Finally, a comment on the range of validity of the analysis is in order: while the couplings studied do not depend on the actual value of $\Lambda_{\mathrm{DM}}$, our results should only be taken as indicative when involving scales $\left(m_{S}\right.$ or $\left.p_{T}\right)$ above $1 \mathrm{TeV}$, as the heavy scale $\Lambda_{\mathrm{DM}}$ cannot plausibly be much larger while still having an impact on the present and foreseen experimental sensitivities.

\subsection{Dark Matter relic density}

Assuming that the singlet scalar particle $S$ is a thermal relic, its abundance $\Omega_{S}$ today is determined by the thermally averaged annihilation cross section into SM particles in the early Universe $(\sigma \mathrm{v})_{\text {ann }}=\sigma(S S \rightarrow X X) \mathrm{v}$. For non-relativistic relics, this cross section can be expanded as

$$
(\sigma \mathrm{v})_{\mathrm{ann}}=\alpha_{s}+\alpha_{p} \mathrm{v}^{2}
$$

where $\alpha_{s}$ is the (unsuppressed) $s$-wave contribution, and the next order in the expansion, $\alpha_{p}$, corresponds to the $p$-wave contribution. Noticing that $\langle\mathrm{v}\rangle^{2}=6 / x_{F}$, with $x_{F}$ given by the freeze-out temperature as $x_{F}=m_{S} / T_{F} \simeq 20$, the relic density is determined by

$$
\Omega_{S} h^{2} \simeq \frac{2.09 \times 10^{8} \mathrm{GeV}^{-1}}{M_{P} \sqrt{g_{* s}\left(x_{F}\right)}\left(\alpha_{s} / x_{F}+3 \alpha_{p} / x_{F}^{2}\right)},
$$

with $M_{P}$ being the Planck mass and $g_{* s}\left(x_{F}\right)$ the number of relativistic degrees of freedom at a temperature $T_{F}$. The $s$-wave contributions to the DM annihilation cross-section for the different channels (the corresponding tree-level Feynman diagrams are shown in appendix B) are given by

$$
\begin{aligned}
\alpha_{s}(S S \rightarrow f \bar{f})= & \frac{6 \lambda_{S}^{2}}{\pi m_{S}^{2}} \frac{r_{f}^{2}\left(1-r_{f}^{2}\right)^{3 / 2}}{\left(r^{2}-4\right)^{2}}\left[\left(1+\frac{4 c_{2} a_{2}}{r_{v}^{2}}\right)^{2}+\frac{c_{4}^{2}}{r_{v}^{4}} \frac{\left(r^{2}-4\right)^{2}}{1-r_{f}^{2}}\right] \\
\alpha_{s}(S S \rightarrow h h)= & \frac{\lambda_{S}^{2}}{8 \pi m_{S}^{2}} \frac{\sqrt{1-r^{2}} K_{h_{0}}^{2}}{\left(r^{4}-6 r^{2}+8\right)^{2}}\left[1+\frac{4 c_{2} a_{2}}{K_{h_{0}}} \frac{r^{2}}{r_{v}^{2}}\left(4 r_{v}^{2}\left(r^{2}-4\right)-3\left(r^{2}-2\right)+\right.\right. \\
& \left.\left.+2 c_{2} a_{2} r^{2}\left(r^{2}-4\right)+\frac{b_{2}}{a_{2}} \frac{r^{4}-6 r^{2}+8}{r^{2}}\right)\right]^{2} \\
\alpha_{s}(S S \rightarrow Z Z)= & \frac{\lambda_{S}^{2}}{8 \pi m_{S}^{2}} \frac{\sqrt{1-r_{Z}^{2}}}{\left(r^{2}-4\right)^{2}} K_{Z_{0}}\left[1+\frac{4 c_{2} a_{2}}{r_{v}^{2}}+\left(c_{1}+2 c_{3}\right) \frac{r^{2}-4}{r_{v}^{2}}\right]^{2} \\
\alpha_{s}\left(S S \rightarrow W^{+} W^{-}\right)= & \frac{\lambda_{S}^{2}}{4 \pi m_{S}^{2}} \frac{\sqrt{1-r_{W}^{2}}}{\left(r^{2}-4\right)^{2}} K_{W_{0}}\left[1+\frac{4 c_{2} a_{2}}{r_{v}^{2}}+c_{1} \frac{r^{2}-4}{r_{v}^{2}}\right]^{2} \\
\alpha_{s}(S S \rightarrow Z h)= & \frac{\lambda_{S}^{2}}{512 \pi m_{S}^{2}} \frac{\left[\left(r^{2}+r_{Z}^{2}-4\right)^{2}-4 r^{2} r_{Z}^{2}\right]^{3 / 2}}{r_{v}^{4}}\left(2 c_{4}+c_{5} a_{5}\right)^{2}
\end{aligned}
$$


with $r=m_{h} / m_{S}, r_{f}=m_{f} / m_{S}, r_{Z, W}=m_{Z, W} / m_{S}, r_{v}=\sqrt{\lambda_{S}} v / m_{S}$ and $K_{h_{0}}, K_{Z_{0}}, K_{W_{0}}$ defined as

$$
\begin{aligned}
& K_{h_{0}}=(b-3) r^{4}-6(b-1) r^{2}+8 b+8\left(r^{2}-4\right) r_{v}^{2} \\
& K_{Z_{0}}=4\left(1-r_{Z}^{2}\right)+3 r_{Z}^{4} \\
& K_{W_{0}}=4\left(1-r_{W}^{2}\right)+3 r_{W}^{4} .
\end{aligned}
$$

Each annihilation channel contains, in general, new non-linear pieces in addition to the standard contributions, including the decorrelations from $b$ in the $S S \rightarrow h h$ channel. The sole exception to this behaviour is the annihilation channel $S S \rightarrow Z h$, which receives contributions from the CP-violating operators $\mathcal{A}_{4,5}$ and is absent in the standard case, inducing an $s$-wave leading term proportional to $c_{4,5}^{2}$.

In the following we discuss how non-linear contributions change the predictions for the Higgs portal. In a conservative approach, we require the abundance of $S$ particles today not to exceed the total DM density measured by Planck [65], imposing $\Omega_{S} h^{2} \leq \Omega_{\mathrm{DM}} h^{2} \simeq 0.12$ but not requiring $S$ to account for the entire DM relic abundance. ${ }^{3}$ Let us start by discussing the non-linear mismatch between the terms which are linear and quadratic in Higgs fields, parametrised by the coefficient $b$ in eq. (2.10). Values $b \neq 1$ modify the relative strength of the $S S h h$ and $S S h$ couplings w.r.t. the standard Higgs portal. This mismatch can be observed in the region $m_{S}>m_{h}$, where the annihilation into two Higgs bosons is important. As shown in figure 1 , for $b>1$ the annihilation cross section into Higgses increases significantly, thus enlarging the allowed region of parameter space for the non-linear portal.

Consider now the impact of the non-linear $\mathcal{A}_{i}$ operators on $\sigma_{\text {ann }}$. Operators $\mathcal{A}_{1-5}$ affect DM annihilations into gauge bosons, Higgses and $b$-quarks, as shown in appendix B. This modifies the relic density $\Omega_{S}$ both for large and small values of $m_{S}$. To illustrate these new effects, we compare in figure 2 the parameter space excluded for the standard Higgs portal (our results for the standard Higgs portal scenario are in agreement with those of refs. [73-76]) and in the presence of the custodially-preserving and CP-even operators $\mathcal{A}_{1}$ and $\mathcal{A}_{2}$, with $c_{1}, c_{2}$ in the range $[-1,1]$. It shows the drastic increase resulting in the parameter space for DM masses larger than tens of $\mathrm{GeV}$, as compared with the allowed region for the standard portal above the black curve. For simplicity, in this figure the dependence on the Higgs field is fixed to be $\mathcal{F}_{1}(h)=\mathcal{F}_{2}(h)=(1+h / v)^{2}$, corresponding to $a_{1}=b_{1}=a_{2}=b_{2}=1$; we have checked that varying these values does not change noticeably the impact on the Dark Matter relic density $\Omega_{S} h^{2}$, as expected. ${ }^{4}$

In the presence of $\mathcal{A}_{1}, \mathrm{DM}$ can directly interact with $\mathrm{SM}$ gauge bosons via the vertices $S S Z Z$ and $S S W^{+} W^{-}$. The new interactions induced by $\mathcal{A}_{1}$ do not modify the allowed

\footnotetext{
${ }^{3}$ This constitutes another important difference with the analysis of ref. [56], which requires the scalar singlet $S$ to constitute all the DM. Although a direct comparison of our results with those of ref. [56] is then difficult due to the different analysis methodology, we can state that our conclusions are compatible with theirs.

${ }^{4} a_{1}\left(b_{1}\right)$ parametrises vertices $S S V V h(S S V V h h)$, with $V=Z, W^{ \pm}$, whose tree-level contribution to the DM annihilation cross section is very much suppressed due to phase space considerations; a variation of $a_{2}$ can be reabsorbed in the normalisation of $c_{2}$; finally, $b_{2}$ enters the $S S \rightarrow h h$ cross-section for masses $m_{S}>m_{h}$, but its effect is only significant for unrealistically large values of $b_{2}$.
} 


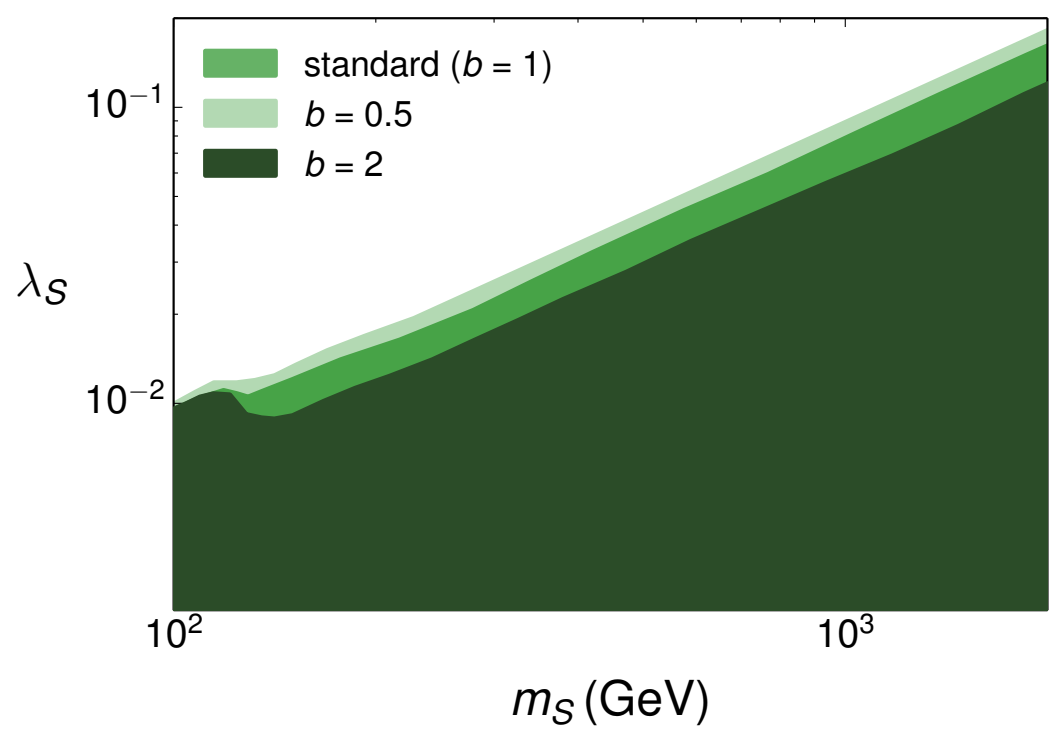

Figure 1. Regions excluded by the condition $\Omega_{S} h^{2} \leq 0.12$ for DM masses $m_{S} \geq 100 \mathrm{GeV}$. The medium green region corresponds to the standard Higgs portal case $b=1$, while the light/dark green regions (superimposed) correspond respectively to $b=0.5$ and $b=2$.
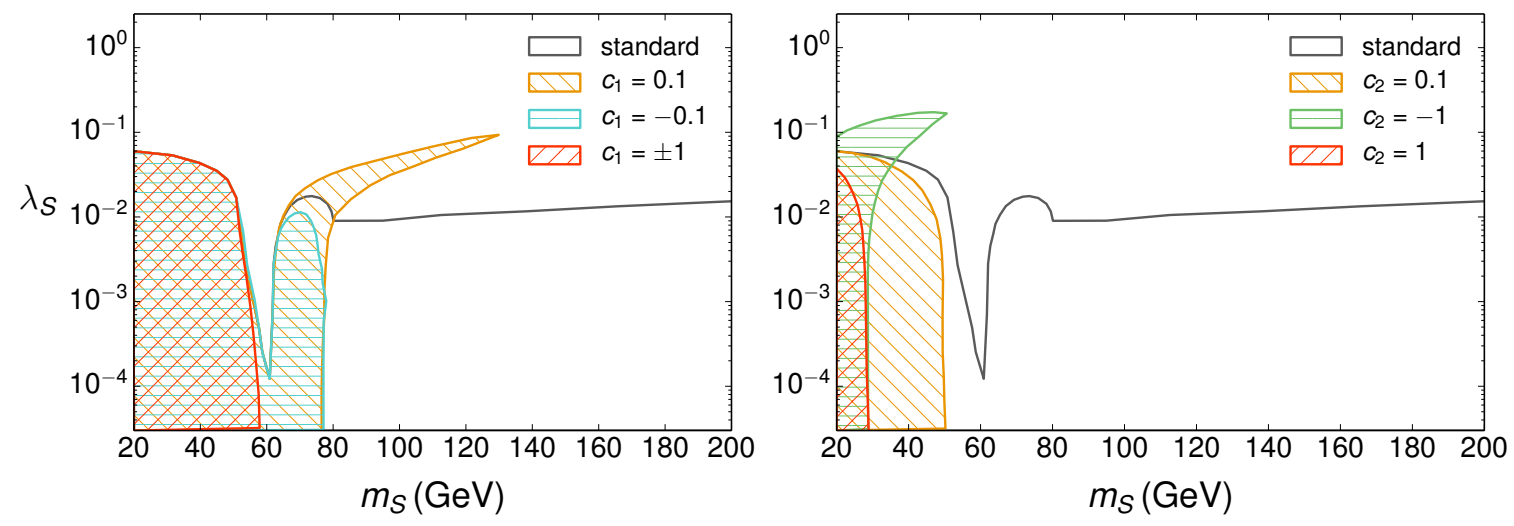

Figure 2. Regions in the $\left(m_{S}, \lambda_{S}\right)$ plane excluded by the constraint $\Omega_{S} h^{2} \leq 0.12$ from Planck [65], in presence of non-linear operators $\mathcal{A}_{1}$ (left) and $\mathcal{A}_{2}$ (right) with $c_{i} \neq 0$. The region below the black line is excluded for the standard Higgs portal. Left: excluded regions for $c_{1}=0.1$ (yellow), $c_{1}=-0.1$ (light blue), $\left|c_{1}\right|=1$ (red). Right: excluded regions for $c_{2}=0.1$ (yellow), $c_{2}=1$ (red), $c_{2}=-1$ (green).

parameter space for $m_{S} \lesssim 65 \mathrm{GeV}$, where DM annihilates dominantly into $b \bar{b}$, while they have a strong impact on the DM annihilation process into two gauge bosons, which becomes important as $m_{S}$ grows, as shown in figure 2 (left). For negative values of $c_{1}$, the positive interference with the linear amplitude (see the Feynman rules in appendix A) increases the total annihilation cross-section everywhere and some of the points ruled out in the standard Higgs portal scenario become viable. On the other hand, if $c_{1}>0$ the interference is destructive and spurious cancellations may happen in regions of the parameter space that are allowed for standard Higgs portals, but become now excluded. As an example, the yellow "branch" structure in figure 2 (left) for $60 \mathrm{GeV} \lesssim m_{S} \lesssim 130 \mathrm{GeV}$ is traversed by a curve on which $\alpha_{s}(S S \rightarrow V V)=0$ for $V=Z, W^{ \pm}$. 
The impact of the operator $\mathcal{A}_{2}$, shown in figure 2 (right), can be understood in an analogous way: the coefficient $c_{2}$ enters the couplings $S S h h$ and $S S h$, with the double effect of boosting the $S S \rightarrow h h$ process for $c_{2}>0$ and generating local cancellations when $c_{2}<0$ on one side, and also altering the annihilation $S S \rightarrow b \bar{b}$ through an $s$-channel Higgs, which significantly affects the annihilation cross section below $m_{S} \simeq m_{h} / 2$.

The operator $\mathcal{A}_{3}$ has a similar phenomenology to that of $\mathcal{A}_{1}$, although restricted exclusively to DM annihilation into $Z$ bosons (at tree level). However, the presence of $\mathcal{A}_{3}$ is tightly constrained by EW precision data (see the discussion at the end of section 2). As the present bound on $c_{3}$ is already below the foreseen experimental sensitivities we will not further analyze it separately.

\subsection{Direct detection of Dark Matter}

DM interactions with nucleons are probed at direct detection experiments, which provide upper limits on the spin-independent and spin-dependent cross-sections. The scalar $S$ interacts with fermions via the Higgs and, in the non-linear case, via $W^{ \pm}$and $Z$ exchange. The most important constraints in our scenario come from the spin-independent limits, which give an upper bound on the cross section $\sigma_{\mathrm{SI}}$ for scattering of $S$ on nucleons. $S$ may not be the only DM particle, but a member of a new DM sector, and in this case $\Omega_{S}<\Omega_{\mathrm{DM}}$. When translating bounds on direct detection cross-section one can account for this fact by the following rescaling

$$
\sigma_{\mathrm{SI}}(S N \rightarrow S N) \frac{\Omega_{S}}{\Omega_{\mathrm{DM}}} \leq \sigma_{\exp }^{\lim },
$$

where $\sigma_{\exp }^{\lim }$ is the experimental upper limit on the DM-nucleon scattering cross-section. Here we consider the current most stringent $95 \%$ Confidence Level (C.L.) experimental limits by LUX [66], as well as the 95\% C.L. projected sensitivity of XENON1T [68].

The white areas in figure 3 and 4 summarise the DM parameter space allowed by Planck data and lying below the XENON1T direct detection sensitivity reach, for the standard and non-linear portals respectively. Specifically, the current and projected direct detection exclusion regions in the plane $\left(m_{S}, \lambda_{S}\right)$ obtained with MicrOMEGAs are shown in figure 3 for the standard Higgs portal scenario, and in figure 4 in the presence of the non-linear operators $\mathcal{A}_{1}$ or $\mathcal{A}_{2}$ with a coefficient $c_{i}=0.1$, fixing for simplicity $\mathcal{F}_{1}(h)=$ $\mathcal{F}_{2}(h)=(1+h / v)^{2}$ (see footnote 4$)$. The following discussion will be restricted to these two cases, that exemplify quite exhaustively the main features introduced by non-linearity. For further scenarios corresponding to different choices of the coefficients $c_{1}, c_{2}$ in the range $[-1,1]$ we defer the reader to appendix C. We stress that, while neither $\mathcal{A}_{1}$ nor $\mathcal{A}_{2}$ affect the $S$-nucleon scattering cross-section to first approximation $\left(\mathcal{A}_{1}\right.$ gives $S S Z Z$ and $S S W^{+} W^{-}$vertices which do not enter the scattering at tree level, while the contribution of $\mathcal{A}_{2}$ is proportional to the transferred momentum, and thus highly suppressed at such low energies), the impact of these two operators on the relic abundance $\Omega_{S}$ affects the direct detection exclusion regions, as shown in figure 4. It is also worth noting that, despite providing an independent and complementary bound to that from the Planck Satellite, the direct detection results share some features with those obtained imposing the constraint 


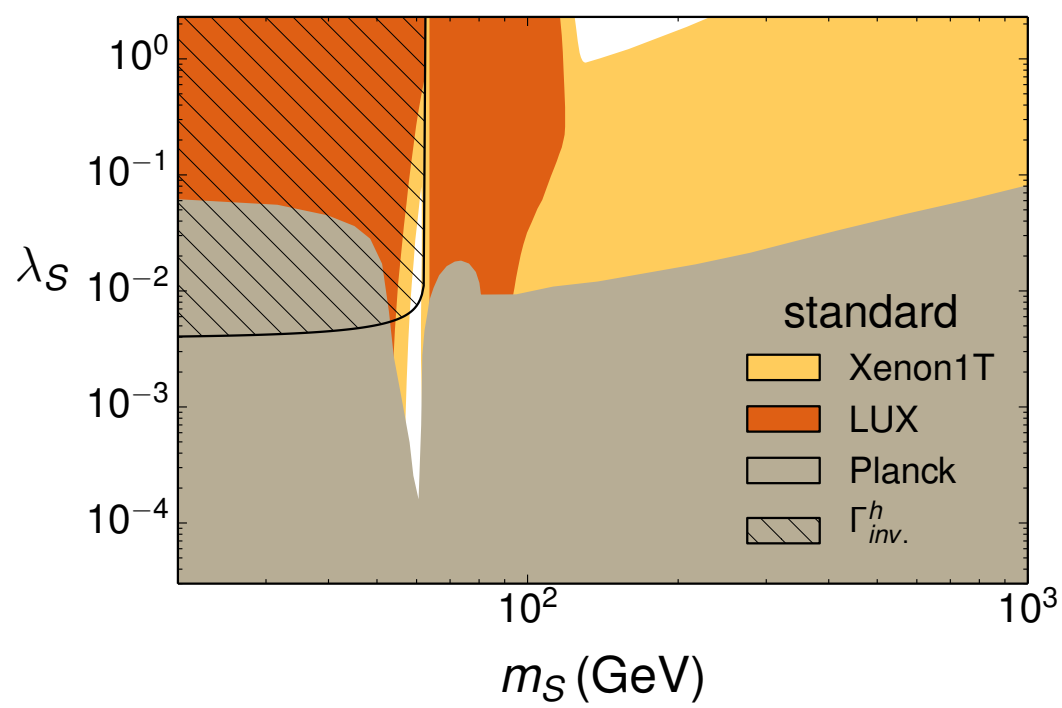

Figure 3. Standard Higgs portal (corresponding to the case $\left.c_{i} \equiv 0, b=1\right)$ in the $\left(m_{S}, \lambda_{S}\right)$ plane, for masses $m_{S}$ up to $1 \mathrm{TeV}$. The grey region is excluded by current bounds from Planck [65]. The orange region is excluded by LUX [66], while the yellow area is currently allowed but within the reach of XENON1T [68]. The black-hatched region represents the region excluded from the invisible Higgs width data (see section 3.3).

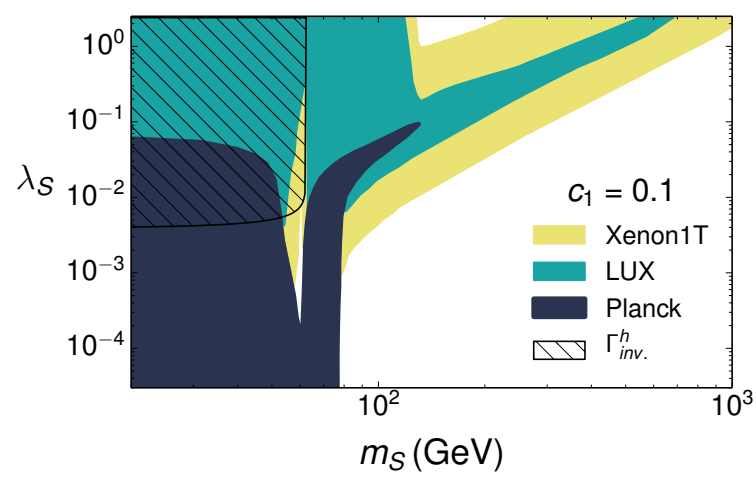

(a) $c_{1}=0.1$

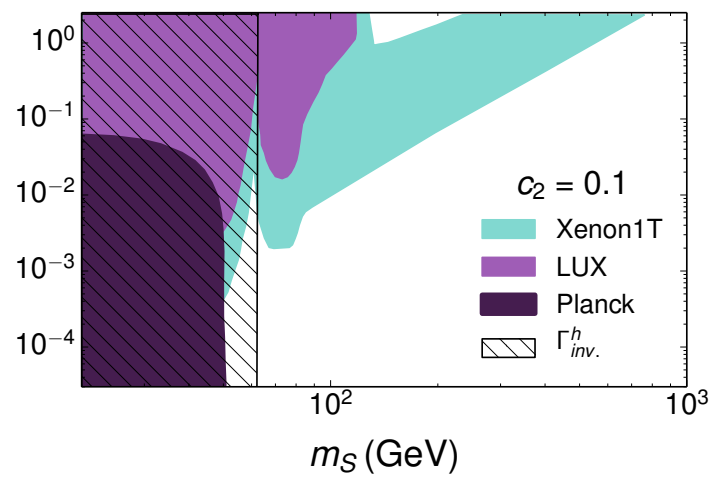

(b) $c_{2}=0.1$

Figure 4. Non-linear Higgs portals in the $\left(m_{S}, \lambda_{S}\right)$ plane, considering the non-linear operators $\mathcal{A}_{1}$ (left) and $\mathcal{A}_{2}$ (right) with $\mathcal{F}_{i}(h)=(1+h / v)^{2}$ and $c_{i}=0.1$. The darkest region is excluded by current bounds from Planck, the green/purple one is excluded by LUX, while the area in yellow/light blue is within the projected reach of XENON1T. The black hatched region represents the bound from the invisible Higgs width (see section 3.3).

by Planck. As discussed in the previous section, the allowed portion of parameter space is generically enlarged for either $c_{1}<0$ or $c_{2}>0$ compared to the standard case (see figure $4 \mathrm{~b}$ ), while for $c_{1}>0$ or $c_{2}<0$ the exclusion region may occasionally stretch further into an area that is allowed in the standard setup, as in figure $4 \mathrm{a}$.

Let us also comment on the impact of the operator $\mathcal{A}_{4}$ on DM-nucleon scattering: as shown in appendix A, this operator induces an effective vertex $S S Z$ that allows a diagram for the $q S \rightarrow q S$ process with a $Z$ boson mediating in $t$-channel. However, the 
corresponding contribution to the squared amplitude is proportional to the Mandelstam variable $t$ :

$$
|A(q S \rightarrow q S)|^{2} \sim c_{4}^{2} \frac{g^{4}}{\left(c_{\theta_{W}}\right)^{4}} \frac{m_{q}^{2}}{m_{Z}^{4}} t
$$

with $c_{\theta_{W}}$ denoting the cosine of the Weinberg angle. This contribution then vanishes in the limit of zero transferred momentum $t \rightarrow 0$. As a result, the coefficient $c_{4}$ is not bounded by direct detection experiments, a conclusion that we have independently verified using MadDM [77].

\subsection{Invisible Higgs decay width}

A very powerful probe of Higgs portal DM in the mass region $m_{S}<m_{h} / 2$ is given by searches for an invisible decay width of the Higgs boson at the LHC. The decay $h \rightarrow S S$ is open for $m_{S}<m_{h} / 2$, and contributes to the Higgs invisible width $\Gamma_{\mathrm{inv}}$ as

$$
\Gamma_{\mathrm{inv}}=\frac{\lambda_{S}^{2} v^{2}}{2 \pi m_{h}} \sqrt{1-\frac{4 m_{S}^{2}}{m_{h}^{2}}}\left(1+\frac{c_{2} a_{2} m_{h}^{2}}{\lambda_{S} v^{2}}\right)^{2}
$$

As is clear from eq. (3.13), the presence of $\mathcal{A}_{2}$ gives a further contribution to $\Gamma_{\text {inv }}$ w.r.t. the standard Higgs portal, such that, if $c_{2} a_{2} \neq 0$, then $\Gamma_{\text {inv }}>0$ even for $\lambda_{S} \rightarrow 0$. Current experimental searches by ATLAS [78, 79] and CMS [80] constrain the $h \rightarrow$ invisible branching fraction, with the strongest limit requiring [79]

$$
\mathrm{BR}_{\mathrm{inv}}=\frac{\Gamma_{\mathrm{inv}}}{\Gamma_{\mathrm{inv}}+\Gamma_{\mathrm{SM}}}<0.23 \quad(95 \% \mathrm{CL})
$$

where the $\mathrm{SM}$ width is $\Gamma_{\mathrm{SM}} \simeq 4 \mathrm{MeV}$. Conveniently setting the parameter $a_{2}=1$ (as it can always be reabsorbed in the normalization of $c_{2}$ ), we present the exclusion region obtained from eqs. (3.13) and (3.14) as a black hatched area in figures 3 and 4a for $c_{2}=0$, and figure $4 \mathrm{~b}$ for $c_{2}=0.1$. For figure $4 \mathrm{a}$ the limit coincides with the one derived for the standard Higgs portal plotted also in figure 3 (see e.g. [73-76]), while figure 4b illustrates the effect of $c_{2} \neq 0$ : even for small values of this coefficient, the bound becomes very stringent, with practically all the region $m_{S}<m_{h} / 2$ being excluded.

It is important to stress that, even though the non-linear operator $\mathcal{A}_{4}$ generates a $S S Z$ vertex, the $Z$ invisible width is not affected by it. The would-be contribution from $\mathcal{A}_{4}$ is $\mathrm{CP}$-odd and also vanishes whenever the $Z$ is on-shell.

The impact of non-linear contributions on the parameter space of Higgs portals, combining the information from the DM relic density, direct detection experiments and searches for invisible decay modes of the Higgs boson is exemplified in figure 5, which shows the comparison between the combined excluded region for the standard Higgs portal (grey region) and the combined excluded regions in the presence of $\mathcal{A}_{1}$ with $c_{1}=0.1$ (hatched-blue region) and in the presence of $\mathcal{A}_{2}$ with $c_{2}=0.1$ (hatched-orange region). 


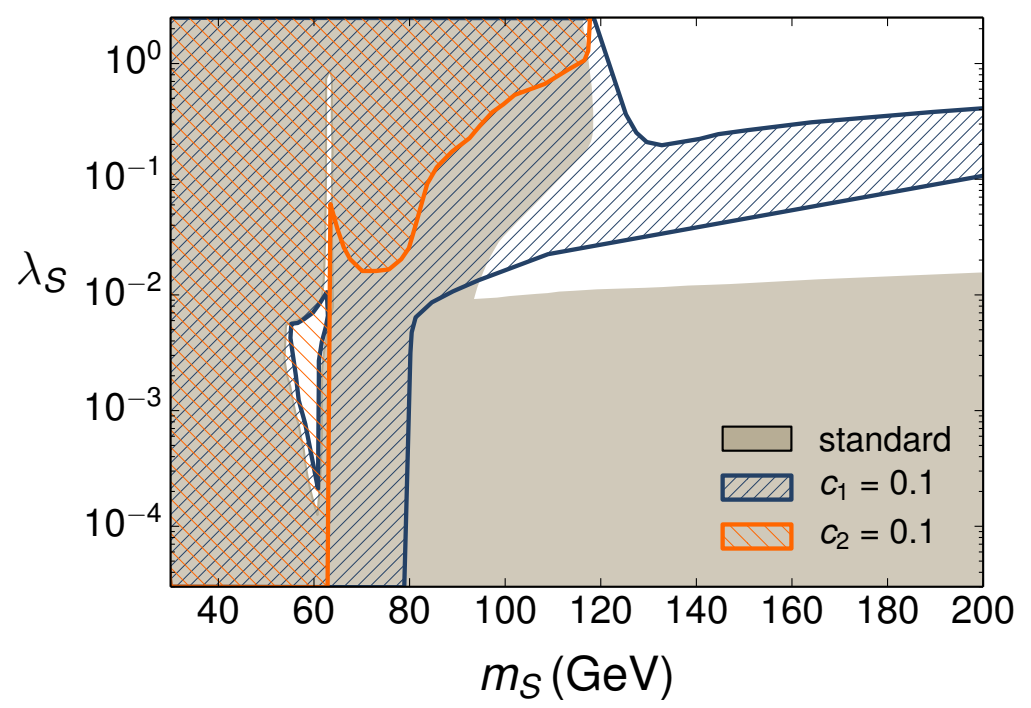

Figure 5. Current excluded region in the $\left(m_{S}, \lambda_{S}\right)$ plane for the standard Higgs portal (grey) versus the non-linear one for $c_{1}=0.1$ (blue) and $c_{2}=0.1$ (orange), from DM relic abundance, direct detection and invisible decay width of the Higgs.

\subsection{Dark Matter at the LHC: mono-X searches}

As already highlighted in the previous section, the LHC (and collider experiments in general) constitutes a natural place to search for DM interactions with the SM, in particular if such interactions involve the EW sector of the theory. LHC probes of DM provide an independent test of the results from low-energy and astrophysical experiments, while being able to directly explore a new energy regime.

A key probe of DM production at colliders are "mono- $X$ " signatures, i.e. the associated production of DM particles with a visible object $X$, which is seen to recoil against a large amount of missing transverse energy $\mathbb{E}_{T}$. These signatures are in principle sensitive to relatively large DM masses, but for the standard Higgs portal scenario the relevant cross sections at the LHC drop very quickly for $m_{S}>m_{h} / 2$, making it challenging to extract information on the DM properties from these searches (see e.g. [25]). As we show below, the presence of non-linear Higgs portal interactions $\mathcal{A}_{1-5}$ has a dramatic impact on the LHC potential for probing such mono- $X$ signatures.

We focus our analysis on mono- $h$, mono- $Z$ and mono- $W$ signatures at the LHC, and present a detailed comparison of the standard and non-linear Higgs portal DM scenarios in this context. We stress that for the case of mono- $h, Z$ signatures, both $\bar{q} q$ and gluon $(g g)$ initiated processes are possible. The latter are characterised by loop-induced DM production processes, which we compute using the FeynRules/NLOCT framework [81] interfaced to MadGraph5_aMC@NLO and MadLoop [82, 83], to ensure that the momentum dependence of the loop is accurately described. This particular aspect is crucial for a meaningful comparison between the standard and non-linear Higgs portal scenarios. 

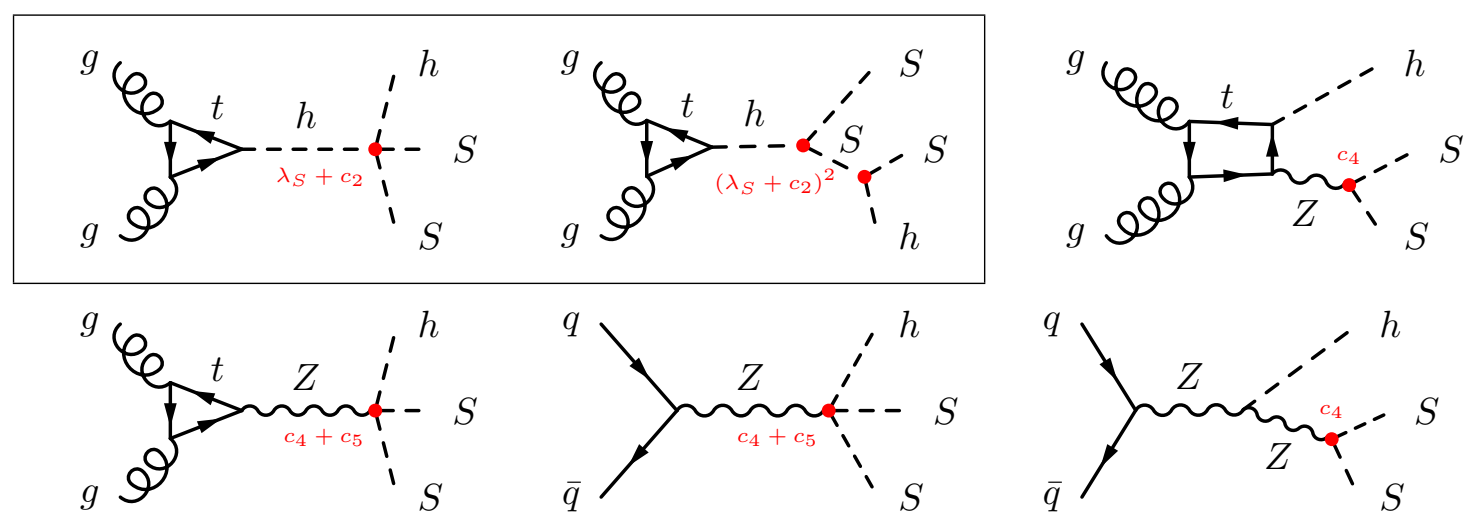

Figure 6. Sample of the main Feynman diagrams contributing to mono- $h$ production. In the standard Higgs case only those inside the frame are present: the process is entirely $g g$-initiated, with contributions proportional to $\lambda_{S}$ and to $\lambda_{S}^{2}$. In the non-linear scenario all the diagrams contribute: both $g g$ - and $\bar{q} q$-initiated processes are included. The proportionality of each diagram to the non-linear parameters is indicated in the figure (overall factors and numerical coefficients are not specified).

\subsubsection{Mono- $h$ signatures}

Mono-Higgs searches [84-87] have been proposed recently as a probe of the DM interactions with the SM, particularly in the context of Higgs portal scenarios. This proposal has led the ATLAS experiment to perform a search for mono- $h$ signatures in the $\mathbb{E}_{T}+\gamma \gamma$ [88] and $\mathbb{E}_{T}+b \bar{b}[89]$ final states with $20.3 \mathrm{fb}^{-1}$ of $\mathrm{LHC} 8 \mathrm{TeV}$ data. While the latter channel is not conclusive for the case of scalar Dark Matter, the former yields a 95\% C.L. limit on the mono- $h$ fiducial cross section $\sigma_{\text {mono- } h}^{\gamma \gamma} \leq 0.7 \mathrm{fb}$ (with $h \rightarrow \gamma \gamma$ ) after the selection $\mathbb{E}_{T}>90 \mathrm{GeV}$.

For the standard Higgs portal, mono- $h$ processes are $g g$-initiated and the amplitude receives contributions from Feynman diagrams scaling as $\sim \lambda_{S}$ and $\sim \lambda_{S}^{2}$, as depicted in figure 6 (within the frame), the latter providing a significant enhancement in the cross section when $\lambda_{S} \sim 1$. We note however that for $\lambda_{S}=1$, satisfying the direct detection bound from LUX requires $m_{S}>127 \mathrm{GeV}$ (see figure 3), and for that range of masses the mono- $h$ cross section gets suppressed due to the intermediate off-shell Higgs state and the steep fall of the gluon PDF at high $\sqrt{\hat{s}}$. Moreover, limits from the invisible decay width of the Higgs require $\lambda_{S} \lesssim 0.007$ for $m_{S}<m_{h} / 2$ in this scenario (see figure 3 ). Overall, the cross section for mono- $h$ in the standard scalar DM Higgs portal is predicted to be very small.

The presence of non-linear Higgs-DM interactions may significantly change the previous picture, as the suppression factors for the standard scenario can be overcome by the appearance of new production channels - e.g. direct couplings of DM to $Z$-bosons which yield a $\bar{q} q$-initiated mono- $h$ contribution (case of $\mathcal{A}_{4}$ and $\mathcal{A}_{5}$ ) - and/or by the momentum dependence of the $S-h, S-Z$ and $S$ - $h-Z$ interactions (case of $\mathcal{A}_{2}, \mathcal{A}_{4}$ and $\mathcal{A}_{5}$ ). A sample of the Feynman diagrams contributing to mono- $h$ in this case is shown in figure 6. For $\mathcal{A}_{2}$, mono- $h$ is $g g$-initiated, and the amplitude receives contributions from Feynman diagrams scaling as $\sim c_{2}$ and $\sim c_{2}^{2} . \mathcal{A}_{4}$ and $\mathcal{A}_{5}$ yield $g g$ - and $\bar{q} q$-initiated contributions to the mono- $h$ 


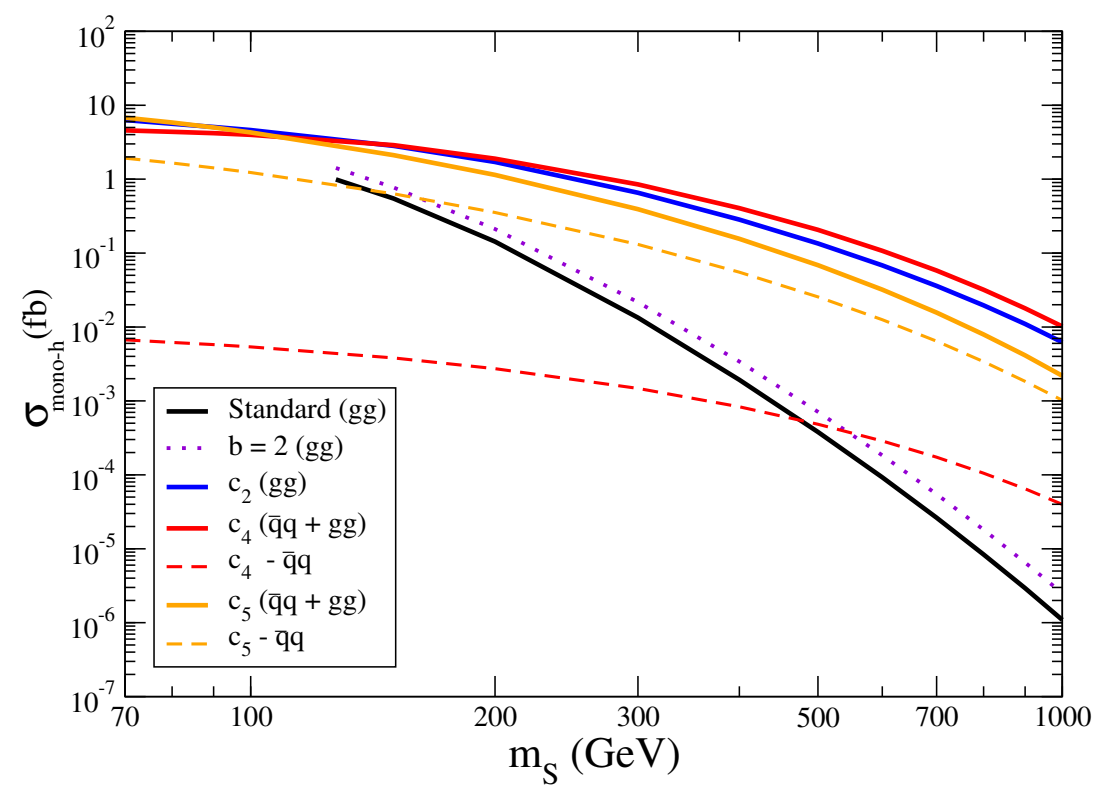

Figure 7. Cross section of the process $p p \rightarrow h S S$ at $\sqrt{s}=13 \mathrm{TeV}$ as a function of $m_{S}$ for the standard Higgs portal with $\lambda_{S}=1$ (solid-black line) and different non-linear setups. The dottedpurple line corresponds to the case $\lambda_{S}=1, b=2, c_{i}=0$. The solid-blue, solid-red and solid-orange lines correspond to $\lambda_{S}=0$ and $c_{2}=1, c_{4}=1, c_{5}=1$ respectively. For the latter two cases, the dashed-red and dashed-orange lines show the $\bar{q} q$-initiated contribution from $\mathcal{A}_{4}$ and $\mathcal{A}_{5}$. The low mass end-point for the solid-black and dotted-purple lines, given by $m_{S}=127 \mathrm{GeV}$, corresponds to the mass bound for the standard Higgs portal scenario for $\lambda_{S}=1$ (see figure 3).

process, both scaling linearly with $c_{4,5}$. In figure 7 we illustrate the behavior of the cross section $\sigma_{\text {mono }-h}=\sigma(p p \rightarrow h S S)$ as a function of the DM mass $m_{S}$ at a centre of mass (c.o.m.) energy of $\sqrt{s}=13 \mathrm{TeV}$, for each of the possible non-linear operators $\mathcal{A}_{i}$ with $c_{i}=1$ and $\lambda_{S}=0$ compared to the standard Higgs portal with $\lambda_{S}=1$ (solid-black line). Let us first note that a non-linear value $b>1$ (dotted-purple line) enhances several processes $\sim \lambda_{S}$ w.r.t. the standard Higgs portal scenario (which modifies the interference between $\sim \lambda_{S}$ and $\sim \lambda_{S}^{2}$ terms) and yields a somewhat larger mono- $h$ cross section. More importantly, figure 7 shows that the presence of either of $\mathcal{A}_{2}$ (solid-blue line), $\mathcal{A}_{4}$ (solid-red line), $\mathcal{A}_{5}$ (solid-orange line) may lead to a large enhancement in the cross section for DM masses $m_{S}>100 \mathrm{GeV}$, potentially reaching enhancements of order $10^{4} \times c_{i}^{2}$ for $m_{S} \gg v$ (we recall that $\lambda_{S}=1$ for the standard Higgs portal scenario is only allowed for $m_{S}>127 \mathrm{GeV}$, and the same bound applies roughly to the scenario $b \neq 1$, as this only has a significant impact on the value of $\Omega_{S}$ for $m_{S}>m_{h}$, as shown in figure 1).

Besides the potentially large increase in the mono- $h$ cross section, in the presence of $\mathcal{A}_{2,4,5}$ the differential distribution of the Higgs boson transverse momentum $P_{T}^{h}$ is shifted towards larger values, as shown in figure 8 for $m_{S}=100 \mathrm{GeV}$ (left) and $m_{S}=500 \mathrm{GeV}$ (right). This much harder mono- $h P_{T}^{h}$ spectrum, particularly for the case of $\mathcal{A}_{5}$, is a landmark signature of non-linear Higgs portals, which also allows for a much better signal extraction from the SM background. 

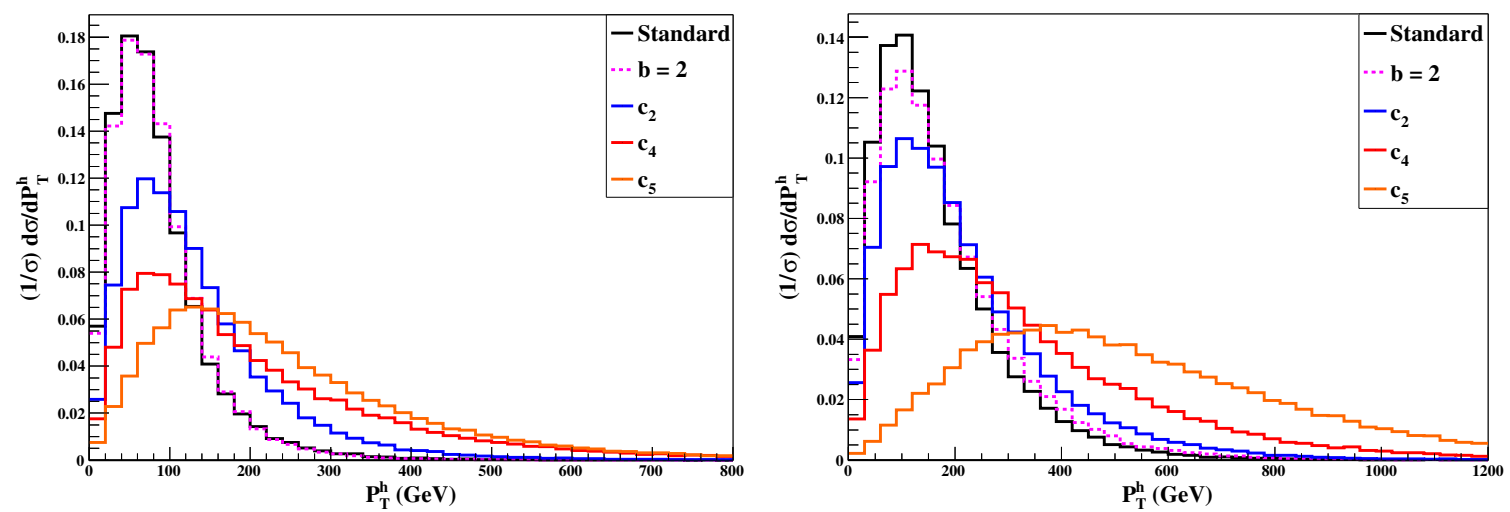

Figure 8. Normalised differential $P_{T}^{h}$ distribution for the process $p p \rightarrow h S S$ in the standard Higgs portal with $\lambda_{S}=1$ (solid-black line), non-linear Higgs portal with $b=2$ (dashed-purple line), $\mathcal{A}_{2}$ with $c_{2}=1$ (solid-blue line), $\mathcal{A}_{4}$ (solid-red line) and $\mathcal{A}_{5}$ (solid-orange line), for $m_{S}=100 \mathrm{GeV}$ (left) and $m_{S}=500 \mathrm{GeV}$ (right).

Finally, let us stress that given the $13 \mathrm{TeV}$ results from figure 7 the $8 \mathrm{TeV}$ mono-Higgs searches at the LHC do not put any meaningful constraint on the parameter space under discussion here, since if we assume a SM value for $\operatorname{Br}(h \rightarrow \gamma \gamma) \simeq 2 \cdot 10^{-3}$ the ATLAS $95 \%$ C.L. limit [88] on the fiducial mono- $h$ cross section is $\sigma_{\text {mono- } h} \leq 0.35 \mathrm{pb}$, two orders of magnitude larger than the $(13 \mathrm{TeV})$ cross sections showed in figure 7 .

\subsubsection{Mono- $\mathrm{Z}$ and mono-W searches}

As a last category of DM observables, we discuss the searches for mono- $W^{ \pm}[90]$ and mono$Z$ [91-94] signatures at the LHC in the context of Higgs portal scenarios. We first focus on the process $p p \rightarrow Z S S$, which receives non-linear contributions from all the effective operators $\mathcal{A}_{i}$ in eq. (2.7). Both for the standard Higgs portal scenario and in the presence of $\mathcal{A}_{1}, \mathcal{A}_{2}, \mathcal{A}_{3}, \mathcal{A}_{4}$ these contributions are both $g g$ - and $\bar{q} q$-initiated, while $\mathcal{A}_{5}$ only gives rise to $g g$-initiated contributions to mono- $Z$. We also note that $\mathcal{A}_{1}$ and $\mathcal{A}_{3}$ give exactly the same contribution to the mono- $Z$ process if $c_{1}=2 c_{3}$ - see appendix A, and furthermore $c_{3} \lesssim 0.1$ is required from EW precision data (recall the discussion at the end of section 2), so in the following we do not explicitly discuss the impact of $\mathcal{A}_{3}$ on mono- $Z$ searches.

In figure 9 (left) we show the LHC cross sections $\sigma(p p \rightarrow Z S S)$ as a function of $m_{S}$ for a c.o.m. energy $\sqrt{s}=13 \mathrm{TeV}$. The solid-black line corresponds to the standard Higgs portal scenario with $\lambda_{S}=1$ (with $\sigma_{\text {mono }-Z}^{\text {standard }} \sim \lambda_{S}^{2}$ ), which decreases quite fast for increasing $m_{S}$. As in the mono- $h$ case (see section 3.4.1), the solid-green, solid-blue, solid-red and solidorange curves respectively correspond to non-linear Higgs portal scenarios with $\lambda_{S}=0$ and $\mathcal{A}_{1}, \mathcal{A}_{2}, \mathcal{A}_{4}$ or $\mathcal{A}_{5}$ being present with $c_{i}=1$. In all the non-linear setups, $\sigma_{\text {mono- } Z}^{i} \sim c_{i}^{2}$, the only exception being $\mathcal{A}_{4}$, which contributes with diagrams scaling both as $c_{4}$ and as $c_{4}^{2}$. As can be seen from figure 9 , these non-linear contributions yield a significantly larger mono- $Z$ cross section compared to the standard Higgs portal for $m_{S} \simeq 100 \mathrm{GeV}$, leading to very large enhancements for $m_{S} \gg v$. As with the mono- $h$ signature, the non-linear operators $\mathcal{A}_{1,2,4,5}$ also affect the differential distribution of the $Z$-boson transverse momentum $P_{T}^{Z}$, yielding a harder mono- $Z P_{T}^{Z}$ spectrum, as can be seen from figure 10 . This effect is 

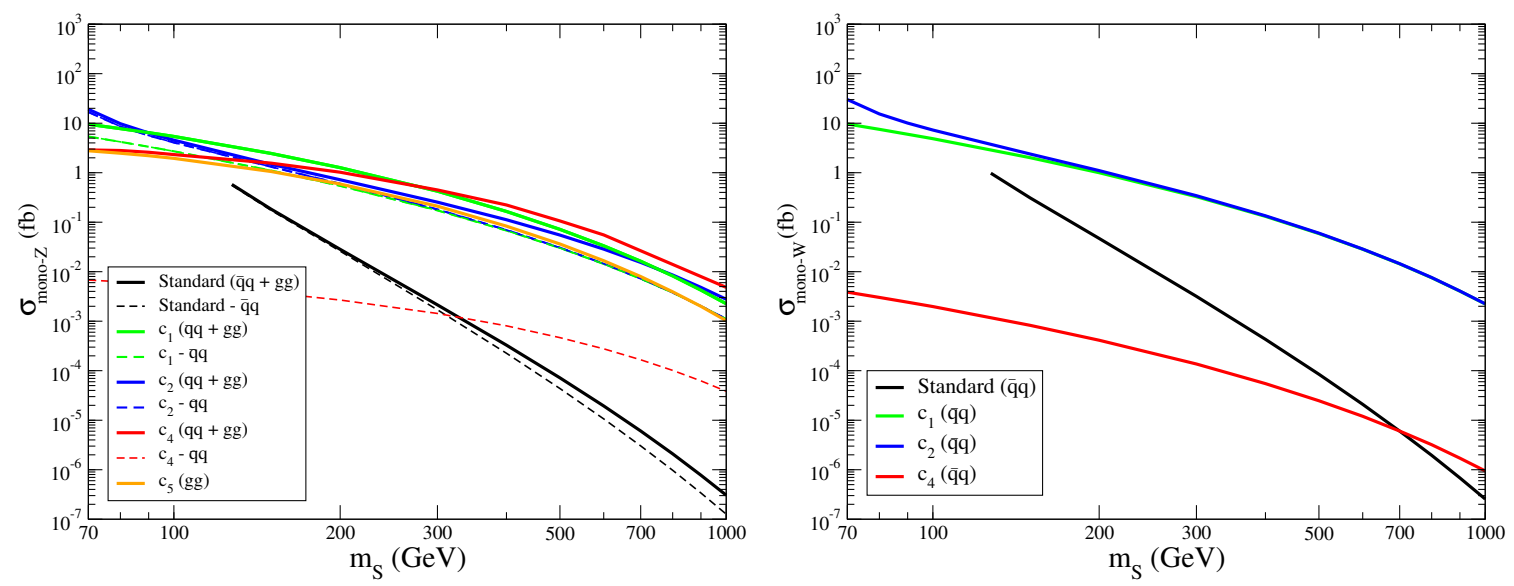

Figure 9. Cross section of the process $p p \rightarrow Z S S$ (left) and $p p \rightarrow W^{ \pm} S S$ (right) at $\sqrt{s}=13 \mathrm{TeV}$ as a function of $m_{S}$ for the standard Higgs portal with $\lambda_{S}=1$ (solid-black line) and different non-linear setups. The solid-green, solid-blue, solid-red and solid-orange lines correspond to $\lambda_{S}=0$ and $c_{1}=1, c_{2}=1, c_{4}=1, c_{5}=1$ respectively. In the left figure, the dashed-black, dashed-green, dashed-blue and dashed-red lines respectively show the $\bar{q} q$-initiated contribution to the process $p p \rightarrow Z S S$ for the standard, $\mathcal{A}_{1}, \mathcal{A}_{2}$ and $\mathcal{A}_{4}$ scenarios.

more important for DM masses in the range 100-300 GeV, while for $m_{S} \gg v$ the standard and non-linear $P_{T}^{Z}$ spectra become very similar. Mono- $Z$ signatures therefore constitute a promising probe of non-linear Higgs portals at the $13 \mathrm{TeV}$ run of the LHC for intermediate DM masses $\left(m_{h} / 2<m_{S} \ll 1 \mathrm{TeV}\right)$ and sizeable values of the coefficients $c_{i} \lesssim 1$. On the other hand, current $8 \mathrm{TeV}$ mono- $Z$ searches at the LHC are only able to constrain values $c_{i} \gg 1$ : the ATLAS analysis [95], using $20.3 \mathrm{fb}^{-1}$ of LHC $8 \mathrm{TeV}$ data, yields $95 \%$ C.L. limits on the mono- $Z\left(Z \rightarrow \ell^{+} \ell^{-}\right)$fiducial cross section $\sigma_{\text {mono- } Z}^{\ell \ell} \leq 2.7 \mathrm{fb}, 0.57 \mathrm{fb}, 0.27 \mathrm{fb}$, $0.26 \mathrm{fb}$ after a corresponding selection $\mathbb{E}_{T}>150 \mathrm{GeV}, 250 \mathrm{GeV}, 350 \mathrm{GeV}, 450 \mathrm{GeV}$. Such limits lie well above the $(13 \mathrm{TeV})$ curves in figure 9 (left), and moreover for fairly light DM $\left(m_{S} \lesssim 100 \mathrm{GeV}\right)$ the selection criteria from the ATLAS search [95] will discard most of the DM signal, as shown in figure 10.

Turning now to mono- $W^{ \pm}$signatures, these are affected by the non-linear operators $\mathcal{A}_{1}$, $\mathcal{A}_{2}$ and $\mathcal{A}_{4}$. Both for these operators and for the standard Higgs portal, the contributions to mono- $W^{ \pm}$are all $\bar{q} q$-initiated, which as we will see makes an important difference w.r.t. the case of mono- $Z$ signatures. In figure 9 (right) we show the cross section $\sigma\left(p p \rightarrow W^{ \pm} S S\right)$ as a function of $m_{S}$ for the standard and non-linear Higgs portal scenarios (using the same criteria and colour convention as for the mono- $Z$ analysis). In the presence of $\mathcal{A}_{1}$ and/or $\mathcal{A}_{2}$ a significant enhancement in the cross section can occur for large values of $m_{S}$, similar to the case of mono- $Z$ and mono- $h$ signatures. However, for the operator $\mathcal{A}_{4}$ mono- $W^{ \pm}$ signatures are very suppressed, as the dominant $g g$-initiated contribution (compare the solid- and dashed-red lines in figure 9 (left) for mono- $Z$ ) is absent in this case. We find that, contrary to the situation encountered in the mono- $h$ and mono- $Z$ analyses above, for mono- $W^{ \pm}$signatures with $W^{ \pm} \rightarrow \ell^{ \pm} \nu_{\ell}$ the $P_{T}^{\ell}$ of the final state lepton has a very similar distribution for the standard and non-linear Higgs portal scenarios, both for low and high DM masses, as seen in figure 11. 

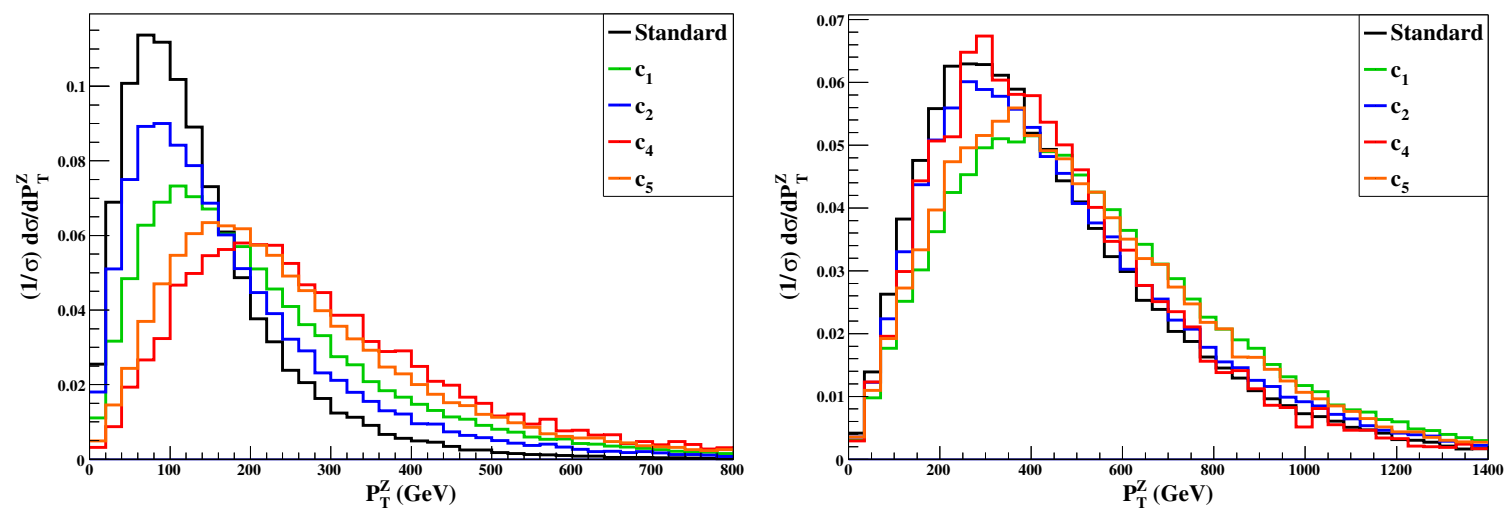

Figure 10. Normalised differential $P_{T}^{Z}$ distribution for the process $p p \rightarrow Z S S$ in the standard Higgs portal with $\lambda_{S}=1$ (black line), and for non-linear Higgs portal operators $\mathcal{A}_{1}$ (green line), $\mathcal{A}_{2}$ (blue line), $\mathcal{A}_{4}$ (red line) and $\mathcal{A}_{5}$ (orange line), for $m_{S}=100 \mathrm{GeV}$ (left) and $m_{S}=500 \mathrm{GeV}$ (right).
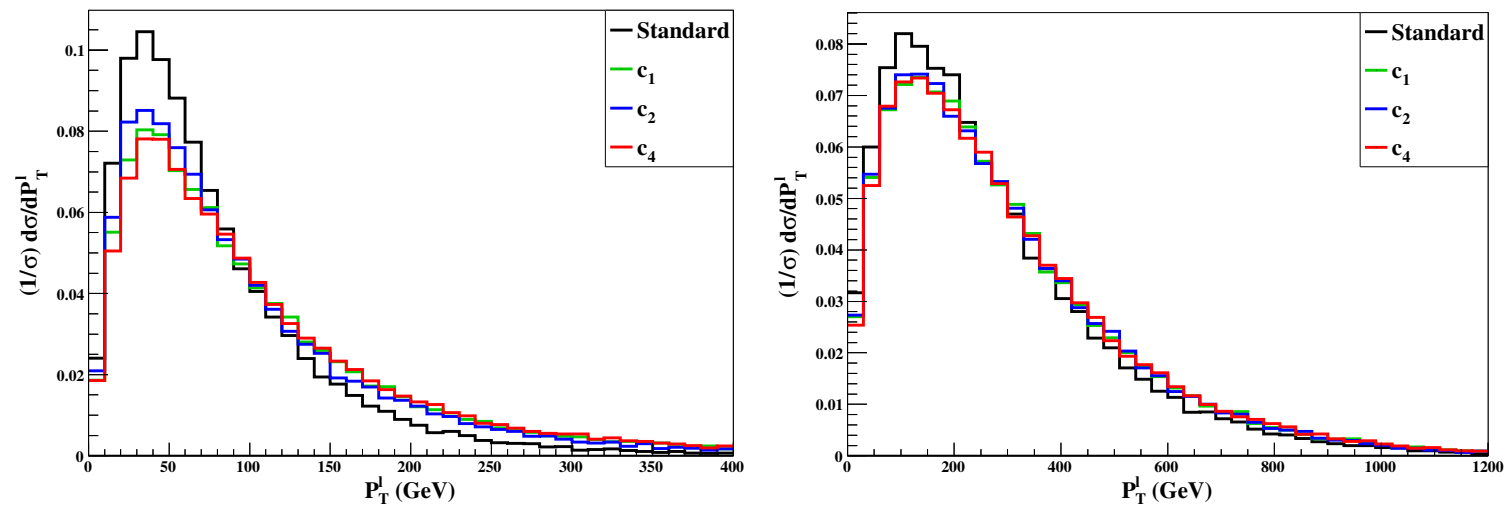

Figure 11. Normalised differential $P_{T}^{\ell}$ distribution for the process $p p \rightarrow W^{ \pm} S S\left(W^{ \pm} \rightarrow \ell^{ \pm} \nu_{\ell}\right)$ in the standard Higgs portal (black line), and for non-linear Higgs portal operators $\mathcal{A}_{1}$ (green line), $\mathcal{A}_{2}$ (blue line) and $\mathcal{A}_{4}$ (red line), for $m_{S}=100 \mathrm{GeV}$ (left) and $m_{S}=500 \mathrm{GeV}$ (right).

Finally, we discuss the possibility of using the ratio $R_{W Z} \equiv \sigma(p p \rightarrow Z S S) / \sigma\left(p p \rightarrow W^{ \pm} S S\right)$ as a probe of non-linear Higgs portal scenarios, as shown in figure 12 (left) as a function of $m_{S}$. Remarkably, the impact of each non-linear operator on this ratio is determined only by its gauge and Lorentz structure, independently of the value of the coefficient ${ }^{5} c_{i}$. Analogously, the dependence on $\lambda_{S}$ factors out in the standard case. While the effect of the operator $\mathcal{A}_{2}$ on this observable cannot be effectively disentangled from that of a standard Higgs portal (as can be seen by comparing the black and blue curves in figure 12 (left)), the ratio $R_{W Z}$ is a very powerful non-linear discriminator for the cases of $\mathcal{A}_{1}$ and $\mathcal{A}_{4}$ (also trivially for $\mathcal{A}_{5}$, for which the mono- $W^{ \pm}$process is absent and $\left.R_{W Z} \equiv \infty\right)$, corresponding respectively to the green and red curves in figure 12 (left). Moreover, recalling that the operator $\mathcal{A}_{3}$ enters the mono- $Z$ process with the corresponding coefficient in the combination $\left(c_{1}+2 c_{3}\right)$ (see appendix A), while it does not enter the mono- $W^{ \pm}$process, the green

\footnotetext{
${ }^{5}$ The line for $\mathcal{A}_{4}$ is an exception, due to the fact that the amplitude for mono- $Z$ receives contributions scaling both as $c_{4}$ and as $c_{4}^{2}$, so that the coefficient does not factor out in $R_{W Z}$. However, this does not impair the interpretation of the plot in figure 12 .
} 

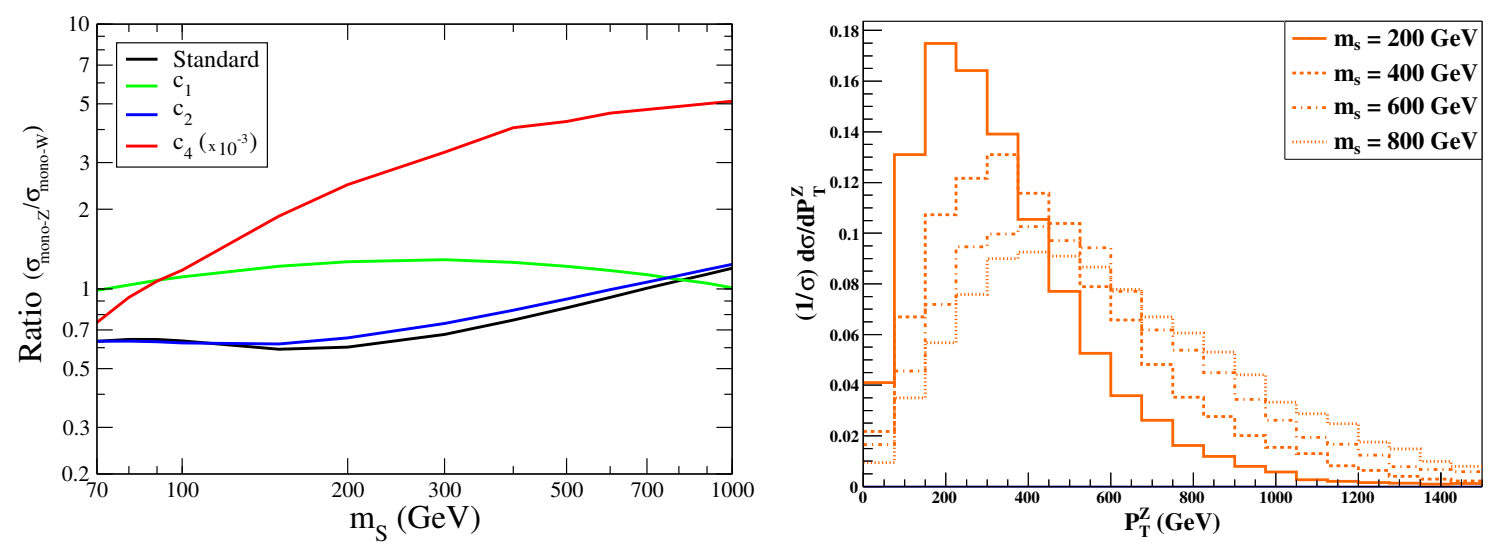

Figure 12. Left: cross section ratio $R_{W Z} \equiv \sigma(p p \rightarrow Z S S) / \sigma\left(p p \rightarrow W^{ \pm} S S\right)$ at $\sqrt{s}=13 \mathrm{TeV}$ as a function of $m_{S}$ in the standard Higgs portal scenario (black line) and for the non-linear operators $\mathcal{A}_{1}$ (green-line), $\mathcal{A}_{2}$ (blue-line) and $\mathcal{A}_{4}$ (red-line), the latter ratio having been multiplied by $10^{-3}$ to be shown in the figure. Right: normalised differential $P_{T}^{Z}$ distributions for the process $p p \rightarrow Z S S$ for $\mathcal{A}_{5}$ and DM masses $m_{S}=200 \mathrm{GeV}$ (solid), $400 \mathrm{GeV}$ (dashed), $600 \mathrm{GeV}$ (dash-dotted) and $800 \mathrm{GeV}$ (dotted).

curve in figure 12 (left) will get rescaled by $\left(c_{1}+2 c_{3}\right)^{2} / c_{1}^{2}$ in the presence of $\mathcal{A}_{3}$. Thus, for $\operatorname{sign}\left(c_{1}\right)=\operatorname{sign}\left(c_{3}\right)$, the green curve actually represents a lower bound on the contribution of $\mathcal{A}_{1}$ and $\mathcal{A}_{3}$ to the ratio $R_{W Z}$.

Importantly, it is in principle possible to infer the DM mass from the mono- $Z /$ mono$W^{ \pm}$processes through the differential information on the $P_{T}^{V}\left(V=W^{ \pm}, Z\right)$ as shown explicitly in figure 12 (right) for the case of $\mathcal{A}_{5}$ (alternatively, the $\mathbb{E}_{T}$ distribution may be used). Taking this into account, the hypothetical observation of mono- $Z$ and mono- $W$ signals would allow to extract at the same time a measurement of $R_{W Z}$ and of $m_{S}$, i.e. to identify a unique point (surrounded by a finite error region) in the parameter space of figure 12 (left). Naively, the further this point lies away from the black line, the more disfavored the standard portal scenario will be. Employing this technique in a more thorough analysis, which would keep all the relevant uncertainties into account, it would be possible to quantify a confidence level for the exclusion of the standard portal. Therefore, the ratio $R_{W Z}$ can be an efficient probe of the nature of the DM portal to the SM. Notice that the non-linear scenario cannot be ruled out by this kind of study, since any point in the $\left(m_{S}, R_{W Z}\right)$ space corresponds to a whole set of combinations of the coefficients $c_{1-5}$.

\subsection{A comment on indirect detection of Dark Matter}

DM annihilation into charged particles (or states further decaying into charged particles), whether $W^{ \pm}$or charged fermions, would result in significant fluxes of gamma-rays, which can be constrained by astrophysical observations, e.g. from the Fermi-LAT Space Telescope. Rather than performing a detailed study of the indirect detection signatures of non-linear Higgs portal DM scenarios (which we defer for the future), we just discuss briefly the impact of such indirect limits on their parameter space, focusing on DM annihilation into $W^{+} W^{-}$ and $b \bar{b}$, which receive contributions from $\mathcal{A}_{1,2,4}$ and $\mathcal{A}_{2,4}$ respectively (see appendix B). We 


\begin{tabular}{|cc|}
\hline$d=6$ & $d=8$ \\
\hline$b \longrightarrow \mathcal{O}_{b} \equiv\left(\Phi^{\dagger} \Phi\right)^{2} S^{2}$ & $\mathcal{A}_{3} \longrightarrow \mathcal{O}_{3} \equiv\left(\Phi^{\dagger} \stackrel{\leftrightarrow}{D_{\mu}} \Phi\right)\left(\Phi^{\dagger} \stackrel{\leftrightarrow}{D^{\mu}} \Phi\right) S^{2}$ \\
$\mathcal{A}_{1} \longrightarrow \mathcal{O}_{1} \equiv D_{\mu} \Phi^{\dagger} D^{\mu} \Phi S^{2}$ & $\mathcal{A}_{5} \longrightarrow \mathcal{O}_{5} \equiv\left(\Phi^{\dagger} \stackrel{\leftrightarrow}{D_{\mu}} \Phi\right) D^{\mu}\left(\Phi^{\dagger} \Phi\right) S^{2}$ \\
$\mathcal{A}_{2} \longrightarrow \mathcal{O}_{2} \equiv \square\left(\Phi^{\dagger} \Phi\right) S^{2}$ & \\
$\mathcal{A}_{4} \longrightarrow \mathcal{O}_{4} \equiv\left(\Phi^{\dagger} \stackrel{\leftrightarrow}{D_{\mu}} \Phi\right) D^{\mu} S^{2}$ & \\
\hline
\end{tabular}

Table 2. Linear siblings of the non-linear operators $\mathcal{A}_{i}$ and of the deviations of the standard Higgs portal coupling.

consider the limits on such DM annihilation channels from measurements of the gamma-ray flux from the Milky Way galactic center [96], which have been shown to be competitive [97] with those derived from other astrophysical sources, such as dwarf galaxies. Using the limits from [97] on the DM annihilation cross-section $(\sigma \mathrm{v})$ ann into $W^{+} W^{-}$and $b \bar{b}$, given respectively by eqs. (3.6) and (3.3), we can potentially derive constraints on $\lambda_{S}$ and/or $c_{i}$ as a function of the DM mass $m_{S}$. After the appropriate rescaling of the indirect DM signal by $\left(\Omega_{S} / \Omega_{\mathrm{DM}}\right)^{2}$, we find that the current limits from [97] do not provide a meaningful constraint on the parameter space under consideration.

\section{Connection with the linear EFT expansion}

In this section the connection between the non-linear scenario analysed in the previous sections and the linear context is discussed. Eq. (1.1) accounts for the only possible renormalisable coupling between the elementary SM Higgs particle and a singlet scalar DM particle (assuming $Z_{2}$ symmetry). Nevertheless, scenarios for BSM electroweak physics can - and often do - correspond to linear realisations of the EWSB mechanism, typical of perturbative completions. A model-independent parametrisation of the new physics for the SM degrees of freedom is then given by higher-dimension operators of mass dimension $\geq 4$, suppressed by inverse powers of the BSM physics scale $\Lambda \gg v$ : a linear operator expansion, in which the $h$ participates via $\Phi$ insertions and thus through a $(v+h)$ functional dependence. The question then arises of the extent up to which the signals determined above for the non-linear DM portal could be mimicked by effective couplings of the linear expansion, that is by eq. (1.1) plus a tower of operators of mass dimension 6,8 etc.

First of all, the couplings of the non-linear Higgs portal, that is, the deviations from the standard portal given by $b \neq 1$ in eq. (1.2) as well as the operators $\mathcal{A}_{1}-\mathcal{A}_{5}$, appear among the dominant couplings of that expansion, while their linear counterparts are not found at the renormalisable level but only at higher orders in the expansion. Indeed, the siblings (lowest dimension operators in the linear expansion which contain at least the same physical couplings) of $\mathcal{A}_{1}, \mathcal{A}_{2}, \mathcal{A}_{4}$ and the linear operator inducing $b \neq 1$ are linear operators of mass dimension $d=6$, while the couplings $\mathcal{A}_{3}$ and $\mathcal{A}_{5}$ would first appear as $d=8$ linear operators. The explicit definition of the linear siblings can be found in table 2 , providing a one-to-one mapping between the linear and the non-linear operators. 
The complete $d=6$ bosonic linear portal describing the interaction with at most two $S$ fields includes, in addition to $\mathcal{O}_{1}, \mathcal{O}_{2}, \mathcal{O}_{4}$ and $\mathcal{O}_{b}$ above, 9 four-derivative couplings: ${ }^{6}$

$$
\begin{array}{ll}
g^{2} S^{2} W_{\mu \nu} W^{\mu \nu} & g^{2} S^{2} W_{\mu \nu} \tilde{W}^{\mu \nu} \\
g^{\prime 2} S^{2} B_{\mu \nu} B^{\mu \nu} & g^{\prime 2} S^{2} B_{\mu \nu} \tilde{B}^{\mu \nu} \\
g g^{\prime} S^{2} B_{\mu \nu} W^{\mu \nu} & g g^{\prime} S^{2} B_{\mu \nu} \tilde{W}^{\mu \nu} \\
g_{s}^{2} S^{2} G_{\mu \nu} G^{\mu \nu} & g_{s}^{2} S^{2} G_{\mu \nu} \tilde{G}^{\mu \nu}
\end{array}
$$

$\square S \square S$

Being four-derivative couplings, these operators would correspond to sub-dominant operators in the non-linear expansion considered here, which includes at most two-derivative operators; they will thus be disregarded in what follows.

As in the case of non-linear expansion, in order to define a complete basis, fermionic structures should also be considered in addition to those in eq. (2.12):

$$
\bar{Q}_{L_{i}} \Phi d_{R_{j}} S^{2}, \quad \bar{Q}_{L_{i}} \tilde{\Phi} u_{R_{j}} S^{2}, \quad \bar{L}_{L_{i}} \Phi e_{R_{j}} S^{2} .
$$

Again, two flavour blind combinations of the two types of chiral fermion structures (eq. (2.12) and (4.2)) may be related to the bosonic operators $\mathcal{O}_{2}$ and $\mathcal{O}_{4}$, respectively. In order to avoid redundancies either the two combinations or the two latter bosonic operators should then be disregarded [64].

The sector of the linear effective Lagrangian containing the siblings of interest for the comparison is then given by

$$
\mathscr{L}_{S}^{\text {linear portal }} \supset \sum_{i=b, 1,2,4} \frac{c_{i}^{L}}{\Lambda_{\mathrm{DM}}^{2}} \mathcal{O}_{i}+\sum_{i=3,5} \frac{c_{i}^{L}}{\Lambda_{\mathrm{DM}}^{4}} \mathcal{O}_{i},
$$

where $c_{i}^{L}$ denote the operator coefficients.

The rationale of the operator expansions calls for their dimensionless parameters to be naturally $\mathcal{O}(1)$, in which case the answer to the question formulated above is obvious: while $\mathcal{A}_{1}-\mathcal{A}_{5}$ may be expected to contribute with similar strength to the couplings in eq. (1.2), the $d \geq 6$ operators of the linear expansion should be suppressed by powers of $v^{2} / \Lambda_{\mathrm{DM}}^{2} \ll 1$ : in other words, the dominant, leading order effects of the linear expansion are expected to reduce exclusively to those of the standard portal in eq. (1.1), in contrast to the plethora of phenomenological consequences of the leading-order non-linear portal.

It could be argued, though, that fine-tunings occur in nature: in a particular model the amplitude of a given leading operator of the linear expansion could be suppressed, or alternatively that of a higher-dimension operator enhanced. In such an hypothetical situation, is there a way to disentangle the origin of a putative signal of the non-linear basis with respect to that from a sibling linear operator? The answer is positive even if the procedure is involved: a further tool is provided by the comparison - for a given type of coupling - between a vertex with no $h$ leg versus one or more additional $h$ legs, because

\footnotetext{
${ }^{6}$ Other bosonic operators are redundant in that they are related via equations of motion or a total derivative; for instance the operator $\partial_{\mu} S \partial^{\mu} S \Phi^{\dagger} \Phi$ can be reabsorbed by field redefinitions.
} 
they are correlated in the linear case and not so in the non-linear one. For instance the Feynman rules in appendix A, and in particular FR.2 vs. FR.6, illustrate that the couplings $S-S-Z$ and $S-S-Z-h$ are correlated. This is not the case in the non-linear scenario, where these couplings are independent of one another. An analogous effect, due to the different orderings of the operators in the two expansions, is visible in FR.4 vs. FR.5: whilst the vertices $S-S-W-W$ and $S-S-Z-Z$ are proportional to each other in the linear description, they are no longer so in the non-linear case. In practice, such an analysis would be challenging from the experimental point of view, as the identification of these specific couplings is not straightforward with the observables considered here.

Note finally that while some apparent decorrelation may still happen in the linear expansion via a fine-tuned combination of couplings of different orders, with enough data on Higgs physics a global analysis should provide enough resolution on the nature of EWSB involved. Furthermore, that nature would also be expected to show up in other BSM couplings not involving the DM particle.

On a different realm, notice that the comparison between the non-linear portal and the $d \geq 6$ in eq. (4.3) implies a trivial relation between the Lagrangian coefficients of the two expansions, when comparing the intensity of the interactions:

$$
c_{i}^{L} \frac{v^{2}}{\Lambda_{\mathrm{DM}}^{2}}=c_{i} \quad \text { for } \quad i=1,2,4, \quad c_{i}^{L} \frac{v^{4}}{\Lambda_{\mathrm{DM}}^{4}}=c_{i} \quad \text { for } \quad i=3,5 .
$$

It is then straightforward to apply to the linear analysis the results in the plots presented in the previous sections for the non-linear scenario. A caveat should be kept in mind, though, given the limits of validity of the linear expansion: because $v / \Lambda_{\mathrm{DM}} \ll 1$, only those examples explored in which the constraint imposed on the analysis translates into a non-linear coefficient $c_{i}^{L}<4 \pi$, and within the region $\Lambda_{\mathrm{DM}}>m_{S}$, should be retained for consistency of the perturbative expansion, as far as no extra exotic light resonance is detected.

Furthermore, note that in the decoupling limit of the two expansions, $\Lambda \rightarrow \infty$ (corresponding to $c_{i} \rightarrow 0$ in the non-linear case), the effects of the operators $\mathcal{A}_{i}(h)$ (and of the $b \neq 1$ deviations) as well as of their linear siblings vanish. Equivalently, the profiles in the figures in the previous sections approach the standard linear DM portal as the values of the coefficients $c_{i}$ (and of the $b$ deviation) get smaller. This can be explicitly seen in figure 15, where the excluded parameter space increases with the coefficient $c_{1}$ getting smaller in absolute value.

\section{Discussion and conclusions}

In this paper we have studied a new, more general scenario of scalar Higgs portals, with electroweak symmetry breaking non-linearly realised. Within this pattern of symmetry breaking, the physical Higgs particle does not behave as an exact $\mathrm{SU}(2)_{L}$ doublet and in general its participation in couplings as powers of $v+h$-characteristic of the SM and also of BSM linear realisations of the Higgs mechanism- breaks down. We have first noticed how this fact automatically transforms the standard scalar Dark Matter Higgs portal and impacts strongly on the relic abundance. We have then comprehensively described the 
non-linear Higgs portal to Dark Matter: the dominant effective couplings - those not explicitly suppressed by any beyond the SM scale - describing the interactions of a scalar singlet Dark Matter particle with the Higgs field when electroweak symmetry is non-linearly realised. A plethora of new couplings appear involving the SM bosonic sector. The new interactions are characterised by

- Direct couplings to gauge bosons: Dark Matter couples to all Higgs degrees of freedom, namely the Higgs and the longitudinal $W^{ \pm}$and $Z$, see eqs. (1.3), (2.4) and (2.7).

- De-correlation of single and double Higgs couplings: the strength of Dark Matter couplings to one- and two-Higgs fields are are de-correlated in non-linear EWSB, see eq. (2.10).

- Novel kinematic features: non-trivial momentum dependence of Dark Matter interactions due to new derivative couplings provides handles to disentangle linear vs. non-linear Higgs portals at colliders. These features can be extracted from the Lagrangian eq. (2.7), and the Feynman rules derived in appendix A.

We have exploited the features of non-linear Higgs portals using information from CMB measurements, Dark Matter direct detection experiments and LHC searches of visible objects recoiling against missing energy. The effect of non-linear interactions on these observables is summarised in table 1.

As a general feature, in presence of non-linearity the space of parameters for Higgs portals is much less constrained than in the standard picture, see figure 5 for the current exclusion limits. In particular, none of the existing bounds limits the region of masses $m_{S}>200 \mathrm{GeV}$ for couplings $\lambda_{S}$ smaller than 1, except for small regions of the parameter space. Only a limited band within this region will be probed by the next generation of direct detection experiments, see figures $4 \mathrm{a}$ and $4 \mathrm{~b}$ for XENON1T [68] prospects.

The viable parameter spaces differ so much between the two scenarios, that it may be possible to single out signals excluding the standard portal. Let us suppose, for example, that Xenon1T observes a DM signal at a mass $m_{S} \simeq 200 \mathrm{GeV}$, measuring a DM-nucleon scattering cross-section with some value $\hat{\sigma}_{\mathrm{SI}}$. In the standard Higgs-portal interpretation, this would give a point in the $\left(m_{S}, \lambda_{S}\right)$ plane: the coupling is uniquely determined by the values of the mass and of the cross-section. In a non-linear portal setting, instead, the measure would translate into a viable vertical line whose size depends on the values assumed for the non-linear coefficients. Now, it may happen that the point in the linear plane falls within a region which is already ruled out (for example by Planck or by some collider constraint), while the line in the non-linear plot is (at least partly) allowed. This kind of signals would represent a strong indication in favour of extra interactions beyond the standard Higgs portal.

Another characteristic aspect of non-linear portals is the enhancement of signal rates at colliders. In this paper we studied production of a pair of DM particles in association with a vector boson or a Higgs. In the standard Higgs portal, the production of DM particles is unique: a Higgs produced in gluon fusion radiating two DM particles. This production is very suppressed for DM heavier or around the Higgs mass, whereas light DM appears 
already excluded by a combination of Higgs invisible width, relic abundance and direct detection constraints. Non-linear interactions allow electroweak production of DM via couplings to vector bosons, leading to mono- $W$, mono- $Z$ and mono-Higgs signatures with rates $\mathcal{O}\left(10^{1-4}\right) \times c_{i}^{2}$ bigger than the standard Higgs. Additionally, these new production modes exhibit specific kinematic features which may help in disentangling standard and non-linear production. We have shown that a smoking gun to distinguish the standard portal from the non-linear one is provided by the combined measurement of the crosssections ratio $R_{W Z}=\sigma(p p \rightarrow Z S S) / \sigma\left(p p \rightarrow W^{ \pm} S S\right)$ with that of $m_{S}$ from transverse momentum distributions.

For comparative purposes between the linear and non-linear expansions, as part of the theoretical analysis we have determined the linear siblings of all couplings studied. We determined the complete basis of purely bosonic $d=6$ operators of the linear realisation and also the subset of linear $d=8$ operators which induce the same physical couplings as those in the non-linear portal, up to two Dark Matter fields. While all operators of the nonlinear portal considered appear at leading order, their siblings are subleading corrections in the linear expansion and their amplitude should be duly suppressed. Nevertheless, we have discussed how to distinguish the impact of both expansions, in case the relative amplitude of a $d \geq 6$ linear operator becomes enhanced due to some fine-tuning. A tool to disentangle the impact of higher-dimension linear operators from the leading non-linear ones may result, in principle, from the analysis of (de)correlations of specific couplings: $S-S-Z$ vs. $S-S-Z-h$ and $S-S-Z-Z$ vs. $S-S-W-W$. Finally, note that the features and bounds obtained in the analysis of the non-linear portal apply equally well to the standard one, except in regions of the parameter space which undergo restrictions due to constraints on the cut-off of the theory.

The search for Dark Matter and the quest for the nature of electroweak symmetry breaking are major present challenges. We have discussed their interplay within an effective approach, in the framework of the Higgs Dark Matter portal.

\section{Acknowledgments}

We thank A. Manohar and E. Jenkins for useful discussions and for reading the manuscript. The work of K.M. and V.S. is supported by the Science Technology and Facilities Council (STFC) under grant number ST/L000504/1. I.B. research was supported by an ESR contract of the EU network FP7 ITN INVISIBLES (Marie Curie Actions, PITN-GA-2011289442). I.B., M.B.G., L.M., R.dR. acknowledge partial support of the European Union network FP7 ITN INVISIBLES, of CiCYT through the project FPA2012-31880 and of the Spanish MINECO's "Centro de Excelencia Severo Ochoa" Programme under grant SEV2012-0249. M.B.G. and L.M. acknowledge partial support by a grant from the Simons Foundation and the Aspen Center for Physics, where part of this work has been developed, which is supported by National Science Foundation grant PHY-1066293. J.M.N. is supported by the People Programme (Marie Curie Actions) of the European Union Seventh Framework Programme (FP7/2007-2013) under REA grant agreement PIEF-GA2013-625809. 


\section{A Feynman rules}

This appendix provides a complete list of the Feynman rules resulting from the non-linear Higgs portal effective Lagrangian, eq. (2.10), computed in unitary gauge and with momenta understood to flow inwards. The right column shows for comparison the Feynman rules for the case of the linear Higgs portal up to $d=6$.

\begin{tabular}{|c|c|c|c|}
\hline & Standard & Non-linear & Linear $d \leq 6$ \\
\hline (FR.1) & $-4 i \lambda_{S} v$ & $-4 i\left(\lambda_{S} v+\frac{c_{2} a_{2} p_{h}^{2}}{v}\right)$ & $-4 i\left(\lambda_{S} v+\frac{2 v c_{2}^{L} p_{h}^{2}}{\Lambda^{2}}\right)$ \\
\hline (FR.2) & - & $\frac{2 g c_{4}}{c_{\theta}} p_{Z}^{\mu}$ & $-\frac{4 v^{2} g c_{4}^{L}}{c_{\theta} \Lambda^{2}} p_{Z}^{\mu}$ \\
\hline (FR.3) & $-4 i \lambda_{S}$ & $-4 i\left(\lambda_{S} b+\frac{c_{2} b_{2}\left(p_{h 1}+p_{h 2}\right)^{2}}{v^{2}}\right)$ & $-4 i\left(\lambda_{S}+\frac{3 v^{2} c_{b}}{2 \Lambda^{2}}+\frac{2 c_{2}^{L}\left(p_{h 1}+p_{h 2}\right)^{2}}{\Lambda^{2}}\right)$ \\
\hline (FR.4) & - & $-\frac{2 i g^{2}\left(c_{1}+2 c_{3}\right)}{c_{\theta}^{2}} g_{\mu \nu}$ & $-\frac{8 v^{2} i g^{2} c_{1}^{L}}{c_{\theta}^{2} \Lambda^{2}} g_{\mu \nu}$ \\
\hline (FR.5) & - & $-2 i g^{2} c_{1} g_{\mu \nu}$ & $-8 \frac{v^{2}}{\Lambda^{2}} i g^{2} c_{1}^{L} g_{\mu \nu}$ \\
\hline (FR.6) & - & $\frac{4 g}{v c_{\theta}}\left(c_{4} a_{4}\left(p_{Z}+p_{h}\right)^{\mu}-c_{5} a_{5} p_{h}^{\mu}\right)$ & $-\frac{8 v g}{\Lambda^{2} c_{\theta}}\left(c_{4}^{L}\left(p_{Z}+p_{h}\right)^{\mu}\right)$ \\
\hline
\end{tabular}




\section{B Contributions to the Dark Matter relic abundance}

The Feynman diagrams contributing to the main Higgs portal DM annihilation processes are shown next. The labels indicate the parameters entering each vertex (see appendix A for signs and numerical factors). $\lambda_{h}$ in 13a stands for the SM Higgs self-coupling.
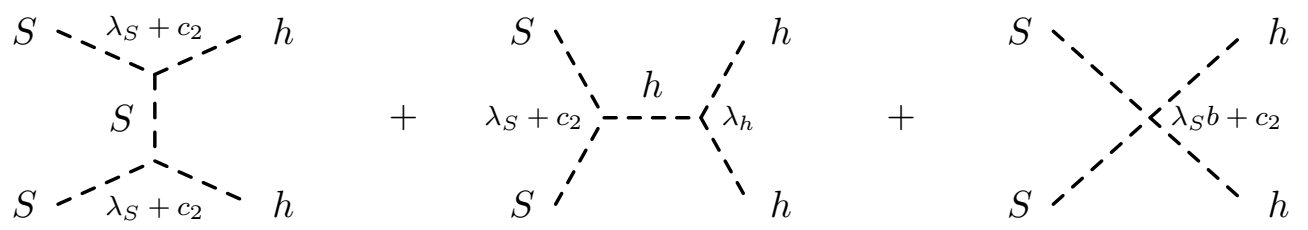

Figure 13 (a). Dark Matter annihilation to Higgs bosons.
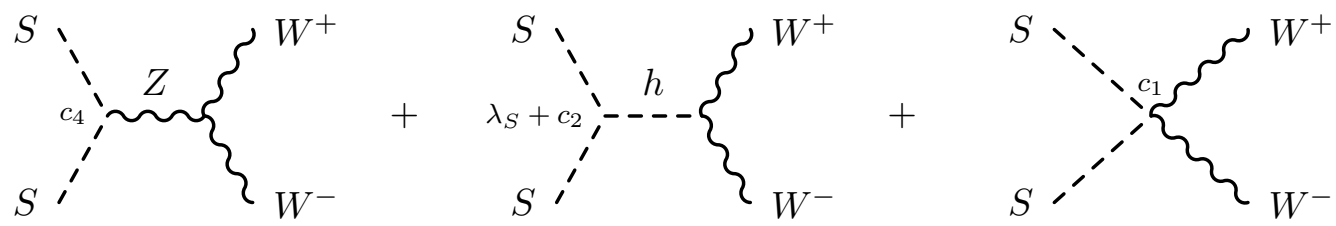

Figure 13 (b). Dark Matter annihilation to $W$ bosons.
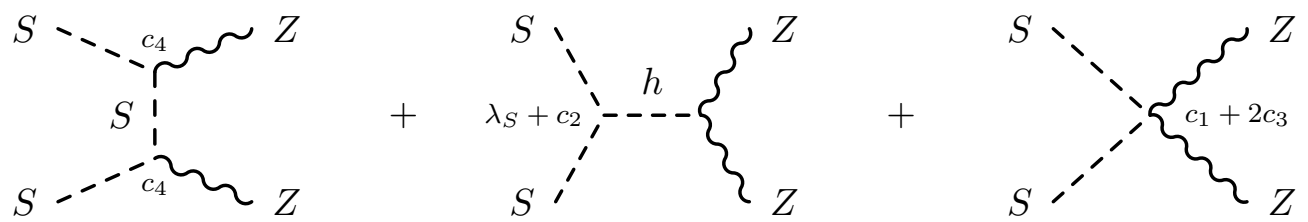

Figure 13 (c). Dark Matter annihilation to $Z$ bosons.
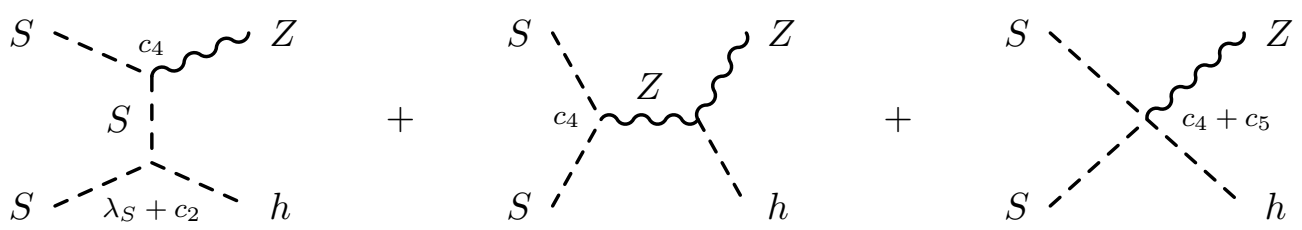

Figure 13 (d). Dark Matter annihilation to $Z$ and Higgs bosons.
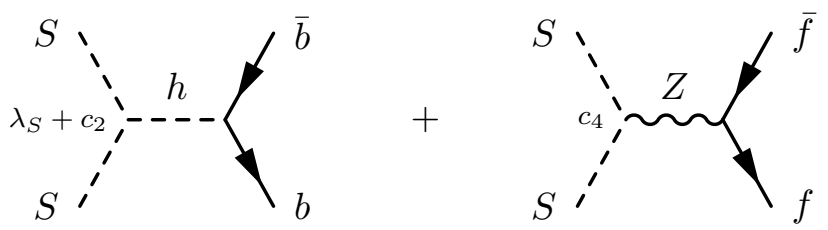

Figure 13 (e). Dark Matter annihilation to $f \bar{f}$. 


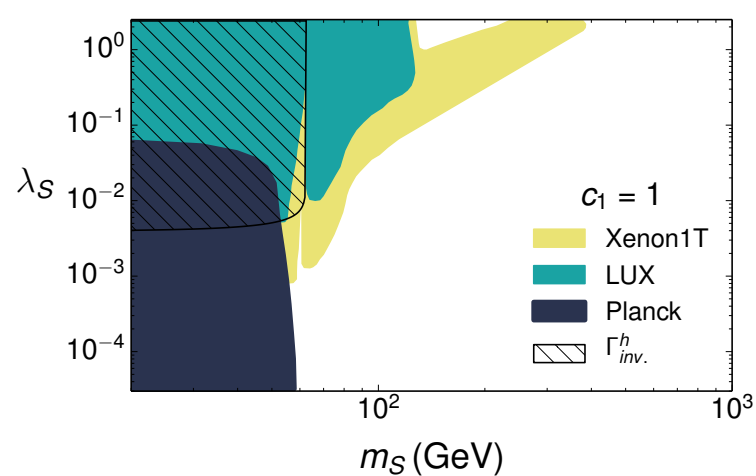

(a) $c_{1}=1$

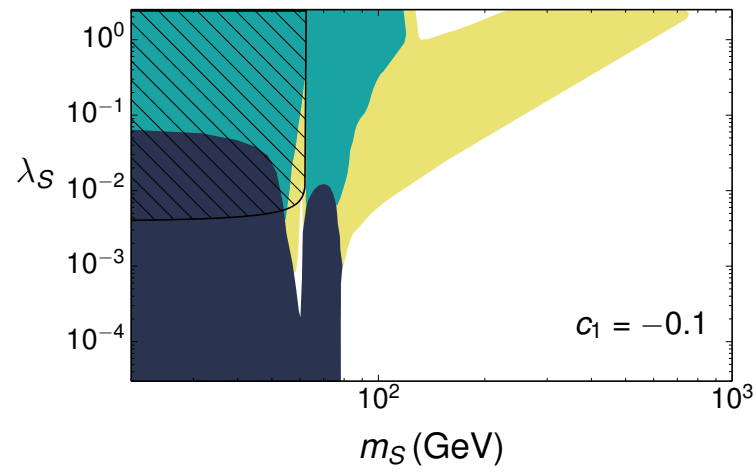

(c) $c_{1}=-0.1$

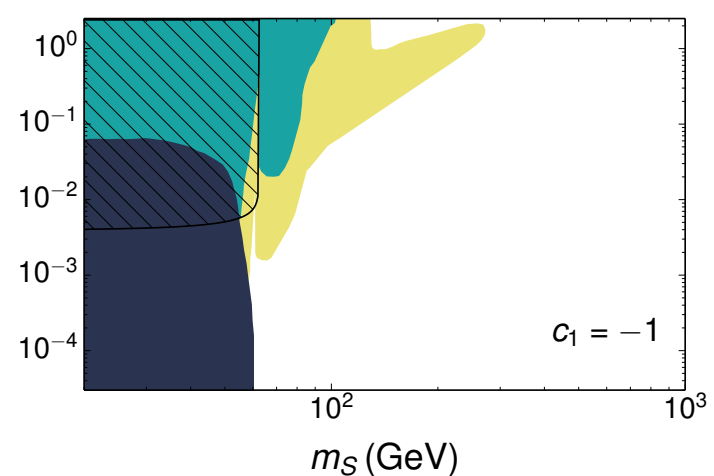

(b) $c_{1}=-1$

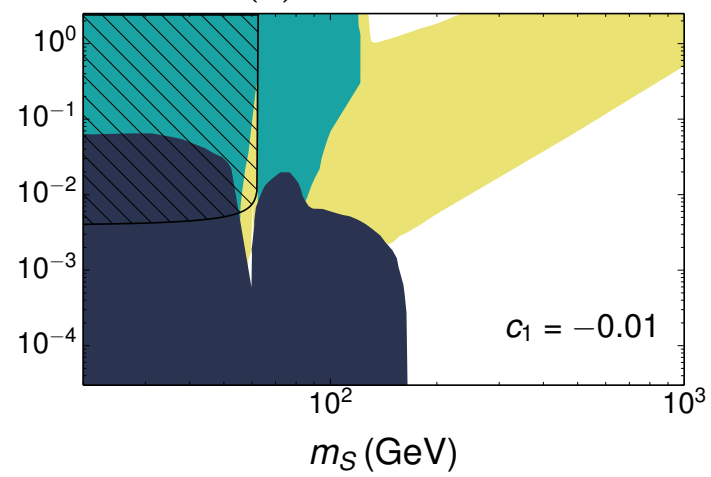

(d) $c_{1}=-0.01$

Figure 14. Results obtained considering the non-linear operator $\mathcal{A}_{1}$ with $\mathcal{F}_{1}(h)=(1+h / v)^{2}$ and for different values of the coefficient $c_{1}$. The blue region is excluded by current bounds from Planck, the green one is excluded by LUX, while the area in yellow is within the projected reach of XENON1T. The black hatched region represents the bound from invisible Higgs width (same as in the linear scenario).

\section{Impact of $\mathcal{A}_{1}$ and $\mathcal{A}_{2}$ for other choices of $c_{i}$}

The analysis of the current constraints on the parameter space of non-linear Higgs portals described in section 3 is restricted to two specific non-linear setups: fixing either $c_{1}$ or $c_{2}$ to 0.1 (see figure 4). Although the main features of non-linearity are quite exhaustively illustrated by these two examples, it is interesting to explore further scenarios, where the coefficients $c_{1}$ and $c_{2}$ are assigned different values in the range $[-1,1]$. In this appendix we show the exclusion regions obtained for $c_{i}=\{ \pm 1,-0.1,-0.01\}$ and $c_{2}= \pm 1$. These figures shall be compared with figure 3 , where the same constraints have been applied to the linear Higgs-portal scenario.

As a general feature, it is worth noticing that in presence of non-linearity, even conveyed by a coefficient of order 0.1 (figures 4 and 14c) or 0.01 (figure 14d), the space of parameters for Higgs portals is much less constrained than in the standard picture. In particular, none of the existing bounds limit the region of masses $m_{S}>200 \mathrm{GeV}$ for couplings $\lambda_{S}$ smaller than 1 , except for small regions of the parameter space. Only a limited band within this region will be probed by the next generation of direct detection experiments (the plots show the reach of XENON1T [68]). 


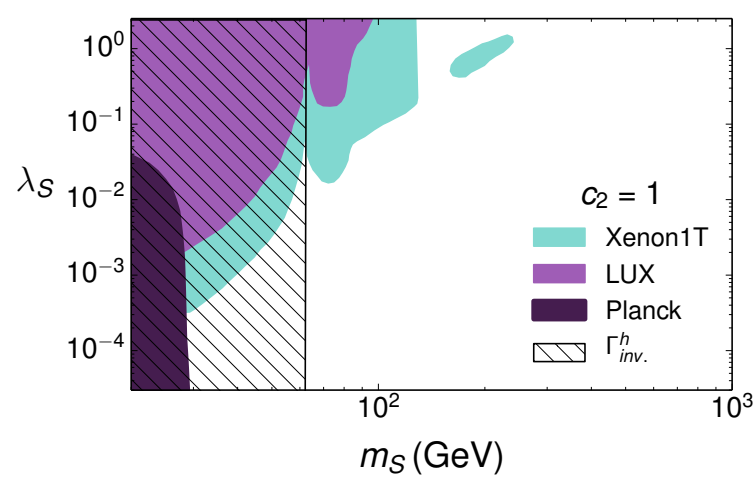

(a) $c_{2}=1$

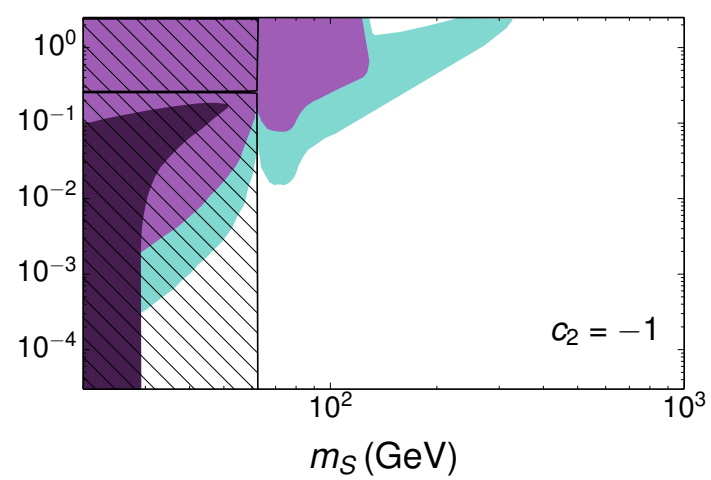

(b) $c_{2}=-1$

Figure 15. Results obtained considering the non-linear operator $\mathcal{A}_{2}$ with $\mathcal{F}_{2}(h)=(1+h / v)^{2}$ and for $c_{2}= \pm 1$. The darkest region is excluded by current bounds from Planck, the purple one is excluded by LUX, while the area in light blue is within the projected reach of XENON1T. The black hatched region represents the bound from invisible Higgs width.

Open Access. This article is distributed under the terms of the Creative Commons Attribution License (CC-BY 4.0), which permits any use, distribution and reproduction in any medium, provided the original author(s) and source are credited.

\section{References}

[1] R. Bernabei et al., Final model independent result of DAMA/LIBRA-phase1, Eur. Phys. J. C 73 (2013) 2648 [arXiv:1308.5109] [INSPIRE].

[2] C. Boehm, J.A. Schewtschenko, R.J. Wilkinson, C.M. Baugh and S. Pascoli, Using the Milky Way satellites to study interactions between cold dark matter and radiation, Mon. Not. Roy. Astron. Soc. 445 (2014) L31 [arXiv: 1404.7012] [INSPIRE].

[3] D. Harvey, R. Massey, T. Kitching, A. Taylor and E. Tittley, The non-gravitational interactions of dark matter in colliding galaxy clusters, Science 347 (2015) 1462 [arXiv: 1503.07675] [INSPIRE].

[4] G. Bertone, D. Hooper and J. Silk, Particle dark matter: Evidence, candidates and constraints, Phys. Rept. 405 (2005) 279 [hep-ph/0404175] [INSPIRE].

[5] V. Silveira and A. Zee, Scalar phantoms, Phys. Lett. B 161 (1985) 136 [inSPIRE].

[6] M.J.G. Veltman and F.J. Yndurain, Radiative corrections to WW scattering, Nucl. Phys. B 325 (1989) 1 [INSPIRE].

[7] B. Patt and F. Wilczek, Higgs-field portal into hidden sectors, hep-ph/0605188 [INSPIRE].

[8] Y.G. Kim and K.Y. Lee, The minimal model of fermionic dark matter, Phys. Rev. D 75 (2007) 115012 [hep-ph/0611069] [INSPIRE].

[9] J. March-Russell, S.M. West, D. Cumberbatch and D. Hooper, Heavy Dark Matter Through the Higgs Portal, JHEP 07 (2008) 058 [arXiv: 0801.3440] [INSPIRE].

[10] Y.G. Kim, K.Y. Lee and S. Shin, Singlet fermionic dark matter, JHEP 05 (2008) 100 [arXiv: 0803.2932] [INSPIRE]. 
[11] M. Ahlers, J. Jaeckel, J. Redondo and A. Ringwald, Probing Hidden Sector Photons through the Higgs Window, Phys. Rev. D 78 (2008) 075005 [arXiv:0807.4143] [InSPIRE].

[12] J.L. Feng, H. Tu and H.-B. Yu, Thermal Relics in Hidden Sectors, JCAP 10 (2008) 043 [arXiv: 0808.2318] [INSPIRE].

[13] S. Andreas, T. Hambye and M.H.G. Tytgat, WIMP dark matter, Higgs exchange and DAMA, JCAP 10 (2008) 034 [arXiv:0808.0255] [INSPIRE].

[14] V. Barger, P. Langacker, M. McCaskey, M. Ramsey-Musolf and G. Shaughnessy, Complex Singlet Extension of the Standard Model, Phys. Rev. D 79 (2009) 015018 [arXiv:0811.0393] [INSPIRE].

[15] M. Kadastik, K. Kannike, A. Racioppi and M. Raidal, EWSB from the soft portal into Dark Matter and prediction for direct detection, Phys. Rev. Lett. 104 (2010) 201301 [arXiv: 0912.2729] [INSPIRE].

[16] S. Kanemura, S. Matsumoto, T. Nabeshima and N. Okada, Can WIMP Dark Matter overcome the Nightmare Scenario?, Phys. Rev. D 82 (2010) 055026 [arXiv:1005.5651] [INSPIRE].

[17] F. Piazza and M. Pospelov, Sub-eV scalar dark matter through the super-renormalizable Higgs portal, Phys. Rev. D 82 (2010) 043533 [arXiv: 1003.2313] [INSPIRE].

[18] C. Arina, F.-X. Josse-Michaux and N. Sahu, A Tight Connection Between Direct and Indirect Detection of Dark Matter through Higgs Portal Couplings to a Hidden Sector, Phys. Rev. D 82 (2010) 015005 [arXiv: 1004.3953] [INSPIRE].

[19] I. Low, P. Schwaller, G. Shaughnessy and C.E.M. Wagner, The dark side of the Higgs boson, Phys. Rev. D 85 (2012) 015009 [arXiv:1110.4405] [INSPIRE].

[20] A. Djouadi, O. Lebedev, Y. Mambrini and J. Quevillon, Implications of LHC searches for Higgs-portal dark matter, Phys. Lett. B 709 (2012) 65 [arXiv:1112.3299] [INSPIRE].

[21] C. Englert, T. Plehn, D. Zerwas and P.M. Zerwas, Exploring the Higgs portal, Phys. Lett. B 703 (2011) 298 [arXiv:1106.3097] [INSPIRE].

[22] J.F. Kamenik and C. Smith, Could a light Higgs boson illuminate the dark sector?, Phys. Rev. D 85 (2012) 093017 [arXiv: 1201.4814] [INSPIRE].

[23] M. Gonderinger, H. Lim and M.J. Ramsey-Musolf, Complex Scalar Singlet Dark Matter: Vacuum Stability and Phenomenology, Phys. Rev. D 86 (2012) 043511 [arXiv:1202.1316] [INSPIRE].

[24] O. Lebedev, On Stability of the Electroweak Vacuum and the Higgs Portal, Eur. Phys. J. C 72 (2012) 2058 [arXiv:1203.0156] [INSPIRE].

[25] N. Craig, H.K. Lou, M. McCullough and A. Thalapillil, The Higgs Portal Above Threshold, JHEP 02 (2016) 127 [arXiv: 1412.0258] [INSPIRE].

[26] A. Manohar and H. Georgi, Chiral Quarks and the Nonrelativistic Quark Model, Nucl. Phys. B 234 (1984) 189 [INSPIRE].

[27] D.B. Kaplan and H. Georgi, SU(2) $\times$ U(1) Breaking by Vacuum Misalignment, Phys. Lett. B 136 (1984) 183 [INSPIRE].

[28] D.B. Kaplan, H. Georgi and S. Dimopoulos, Composite Higgs Scalars, Phys. Lett. B 136 (1984) 187 [INSPIRE]. 
[29] T. Banks, Constraints on $\mathrm{SU}(2) \times \mathrm{U}(1)$ breaking by vacuum misalignment, Nucl. Phys. B 243 (1984) 125 [INSPIRE].

[30] H. Georgi, D.B. Kaplan and P. Galison, Calculation of the Composite Higgs Mass, Phys. Lett. B 143 (1984) 152 [inSPIRE].

[31] H. Georgi and D.B. Kaplan, Composite Higgs and Custodial SU(2), Phys. Lett. B 145 (1984) 216 [INSPIRE].

[32] R. Contino, Y. Nomura and A. Pomarol, Higgs as a holographic pseudoGoldstone boson, Nucl. Phys. B 671 (2003) 148 [hep-ph/0306259] [INSPIRE].

[33] K. Agashe, R. Contino and A. Pomarol, The Minimal composite Higgs model, Nucl. Phys. B 719 (2005) 165 [hep-ph/0412089] [INSPIRE].

[34] R. Contino, L. Da Rold and A. Pomarol, Light custodians in natural composite Higgs models, Phys. Rev. D 75 (2007) 055014 [hep-ph/0612048] [INSPIRE].

[35] B. Gripaios, A. Pomarol, F. Riva and J. Serra, Beyond the Minimal Composite Higgs Model, JHEP 04 (2009) 070 [arXiv:0902.1483] [INSPIRE].

[36] F. Feruglio, The Chiral approach to the electroweak interactions, Int. J. Mod. Phys. A 8 (1993) 4937 [hep-ph/9301281] [INSPIRE].

[37] B. Grinstein and M. Trott, A Higgs-Higgs bound state due to new physics at a TeV, Phys. Rev. D 76 (2007) 073002 [arXiv: 0704.1505] [INSPIRE].

[38] R. Contino, C. Grojean, M. Moretti, F. Piccinini and R. Rattazzi, Strong Double Higgs Production at the LHC, JHEP 05 (2010) 089 [arXiv:1002.1011] [INSPIRE].

[39] A. Azatov, R. Contino and J. Galloway, Model-Independent Bounds on a Light Higgs, JHEP 04 (2012) 127 [Erratum ibid. 1304 (2013) 140] [arXiv:1202.3415] [INSPIRE].

[40] F. Englert and R. Brout, Broken Symmetry and the Mass of Gauge Vector Mesons, Phys. Rev. Lett. 13 (1964) 321 [INSPIRE].

[41] P.W. Higgs, Broken symmetries, massless particles and gauge fields, Phys. Lett. 12 (1964) 132 [INSPIRE].

[42] P.W. Higgs, Broken Symmetries and the Masses of Gauge Bosons, Phys. Rev. Lett. 13 (1964) 508 [INSPIRE].

[43] R. Alonso, M.B. Gavela, L. Merlo, S. Rigolin and J. Yepes, Minimal Flavour Violation with Strong Higgs Dynamics, JHEP 06 (2012) 076 [arXiv: 1201.1511] [INSPIRE].

[44] R. Alonso, M.B. Gavela, L. Merlo, S. Rigolin and J. Yepes, The Effective Chiral Lagrangian for a Light Dynamical "Higgs Particle", Phys. Lett. B 722 (2013) 330 [Erratum ibid. B 726 (2013) 926] [arXiv: 1212.3305] [INSPIRE].

[45] R. Alonso, M.B. Gavela, L. Merlo, S. Rigolin and J. Yepes, Flavor with a light dynamical "Higgs particle", Phys. Rev. D 87 (2013) 055019 [arXiv:1212.3307] [INSPIRE].

[46] I. Brivio et al., Disentangling a dynamical Higgs, JHEP 03 (2014) 024 [arXiv:1311.1823] [INSPIRE].

[47] I. Brivio, O.J.P. Éboli, M.B. Gavela, M.C. Gonzalez-Garcia, L. Merlo and S. Rigolin, Higgs ultraviolet softening, JHEP 12 (2014) 004 [arXiv:1405.5412] [INSPIRE].

[48] M.B. Gavela, J. Gonzalez-Fraile, M.C. Gonzalez-Garcia, L. Merlo, S. Rigolin and J. Yepes, CP violation with a dynamical Higgs, JHEP 10 (2014) 44 [arXiv:1406.6367] [INSPIRE]. 
[49] R. Alonso, I. Brivio, B. Gavela, L. Merlo and S. Rigolin, Sigma Decomposition, JHEP 12 (2014) 034 [arXiv: 1409.1589] [INSPIRE].

[50] I.M. Hierro, L. Merlo and S. Rigolin, Sigma Decomposition: The CP-Odd Lagrangian, JHEP 04 (2016) 016 [arXiv: 1510.07899] [INSPIRE].

[51] G. Isidori and M. Trott, Higgs form factors in Associated Production, JHEP 02 (2014) 082 [arXiv: 1307.4051] [INSPIRE].

[52] G. Buchalla, O. Catà and C. Krause, Complete Electroweak Chiral Lagrangian with a Light Higgs at NLO, Nucl. Phys. B 880 (2014) 552 [arXiv:1307.5017] [InSPIRE].

[53] R. Contino, The Higgs as a Composite Nambu-Goldstone Boson, arXiv:1005.4269 [INSPIRE].

[54] M. Frigerio, A. Pomarol, F. Riva and A. Urbano, Composite Scalar Dark Matter, JHEP 07 (2012) 015 [arXiv: 1204.2808] [INSPIRE].

[55] D. Marzocca and A. Urbano, Composite Dark Matter and LHC Interplay, JHEP 07 (2014) 107 [arXiv: 1404.7419] [INSPIRE].

[56] N. Fonseca, R.Z. Funchal, A. Lessa and L. Lopez-Honorez, Dark Matter Constraints on Composite Higgs Models, JHEP 06 (2015) 154 [arXiv:1501.05957] [INSPIRE].

[57] T. Appelquist and C.W. Bernard, Strongly Interacting Higgs Bosons, Phys. Rev. D 22 (1980) 200 [INSPIRE].

[58] A.C. Longhitano, Heavy Higgs Bosons in the Weinberg-Salam Model, Phys. Rev. D 22 (1980) 1166 [INSPIRE].

[59] A.C. Longhitano, Low-Energy Impact of a Heavy Higgs Boson Sector, Nucl. Phys. B 188 (1981) 118 [INSPIRE].

[60] T. Appelquist and G.-H. Wu, The electroweak chiral Lagrangian and new precision measurements, Phys. Rev. D 48 (1993) 3235 [hep-ph/9304240] [InSPIRE].

[61] J. Yepes, Spin-1 resonances in a non-linear left-right dynamical Higgs context, arXiv:1507.03974 [INSPIRE].

[62] J. Yepes, R. Kunming and J. Shu, CP violation from spin-1 resonances in a left-right dynamical Higgs context, arXiv:1507.04745 [INSPIRE].

[63] I. Brivio, M. Gonzalez-Garcia and L. Merlo, Complete Effective Chiral Lagrangian for a Dynamical Higgs, to appear.

[64] I. Brivio et al., Non-linear Higgs portal to Dark Matter and Flavour Effects, to appear.

[65] Planck collaboration, P.A.R. Ade et al., Planck 2015 results. XIII. Cosmological parameters, arXiv:1502.01589 [INSPIRE].

[66] LUX collaboration, D.S. Akerib et al., First results from the LUX dark matter experiment at the Sanford Underground Research Facility, Phys. Rev. Lett. 112 (2014) 091303 [arXiv: 1310.8214] [INSPIRE].

[67] XENON100 collaboration, E. Aprile et al., Dark Matter Results from 225 Live Days of XENON100 Data, Phys. Rev. Lett. 109 (2012) 181301 [arXiv:1207.5988] [INSPIRE].

[68] XENON1T collaboration, E. Aprile, The XENON1T Dark Matter Search Experiment, Springer Proc. Phys. 148 (2013) 93 [arXiv:1206.6288] [INSPIRE]. 
[69] A. Alloul, N.D. Christensen, C. Degrande, C. Duhr and B. Fuks, FeynRules 2.0 - A complete toolbox for tree-level phenomenology, Comput. Phys. Commun. 185 (2014) 2250 [arXiv: 1310.1921] [INSPIRE].

[70] G. Bélanger, F. Boudjema, A. Pukhov and A. Semenov, MicrOMEGAs4.1: two dark matter candidates, Comput. Phys. Commun. 192 (2015) 322 [arXiv:1407.6129] [INSPIRE].

[71] J. Alwall et al., The automated computation of tree-level and next-to-leading order differential cross sections and their matching to parton shower simulations, JHEP 07 (2014) 079 [arXiv: 1405.0301] [inSPIRE].

[72] O. Mattelaer and E. Vryonidou, Dark matter production through loop-induced processes at the LHC: the s-channel mediator case, Eur. Phys. J. C 75 (2015) 436 [arXiv:1508.00564] [INSPIRE].

[73] J.M. Cline, K. Kainulainen, P. Scott and C. Weniger, Update on scalar singlet dark matter, Phys. Rev. D 88 (2013) 055025 [arXiv:1306.4710] [INSPIRE].

[74] M. Duerr, P. Fileviez Perez and J. Smirnov, Scalar Singlet Dark Matter and Gamma Lines, Phys. Lett. B 751 (2015) 119 [arXiv: 1508.04418] [INSPIRE].

[75] M. Duerr, P. Fileviez Perez and J. Smirnov, Scalar Dark Matter: Direct vs. Indirect Detection, arXiv:1509.04282 [INSPIRE].

[76] H. Han and S. Zheng, New Constraints on Higgs-portal Scalar Dark Matter, JHEP 12 (2015) 044 [arXiv: 1509.01765] [INSPIRE].

[77] M. Backović, A. Martini, O. Mattelaer, K. Kong and G. Mohlabeng, Direct Detection of Dark Matter with MadDM v.2.0, Phys. Dark Univ. 9-10 (2015) 37 [arXiv:1505. 04190] [INSPIRE].

[78] ATLAS collaboration, Search for invisible decays of a Higgs boson using vector-boson fusion in pp collisions at $\sqrt{s}=8 \mathrm{TeV}$ with the ATLAS detector, JHEP 01 (2016) 172 [arXiv: 1508.07869] [INSPIRE].

[79] ATLAS collaboration, Constraints on new phenomena via Higgs boson couplings and invisible decays with the ATLAS detector, JHEP 11 (2015) 206 [arXiv:1509.00672] [INSPIRE].

[80] CMS collaboration, Search for invisible decays of Higgs bosons in the vector boson fusion and associated ZH production modes, Eur. Phys. J. C 74 (2014) 2980 [arXiv:1404.1344] [INSPIRE].

[81] C. Degrande, Automatic evaluation of UV and R2 terms for beyond the Standard Model Lagrangians: a proof-of-principle, Comput. Phys. Commun. 197 (2015) 239 [arXiv:1406.3030] [INSPIRE].

[82] V. Hirschi, R. Frederix, S. Frixione, M.V. Garzelli, F. Maltoni and R. Pittau, Automation of one-loop QCD corrections, JHEP 05 (2011) 044 [arXiv:1103.0621] [INSPIRE].

[83] V. Hirschi and O. Mattelaer, Automated event generation for loop-induced processes, JHEP 10 (2015) 146 [arXiv:1507.00020] [INSPIRE].

[84] A.A. Petrov and W. Shepherd, Searching for dark matter at LHC with Mono-Higgs production, Phys. Lett. B 730 (2014) 178 [arXiv:1311.1511] [InSPIRE].

[85] L. Carpenter, A. DiFranzo, M. Mulhearn, C. Shimmin, S. Tulin and D. Whiteson, Mono-Higgs-boson: A new collider probe of dark matter, Phys. Rev. D 89 (2014) 075017 [arXiv:1312.2592] [INSPIRE]. 
[86] A. Berlin, T. Lin and L.-T. Wang, Mono-Higgs Detection of Dark Matter at the LHC, JHEP 06 (2014) 078 [arXiv: 1402.7074] [INSPIRE].

[87] J.M. No, Looking through the pseudoscalar portal into dark matter: Novel mono-Higgs and mono-Z signatures at the LHC, Phys. Rev. D 93 (2016) 031701 [arXiv:1509.01110] [INSPIRE].

[88] ATLAS collaboration, Search for Dark Matter in Events with Missing Transverse Momentum and a Higgs Boson Decaying to Two Photons in pp Collisions at $\sqrt{s}=8 \mathrm{TeV}$ with the ATLAS Detector, Phys. Rev. Lett. 115 (2015) 131801 [arXiv:1506.01081] [INSPIRE].

[89] ATLAS collaboration, Search for dark matter produced in association with a Higgs boson decaying to two bottom quarks in pp collisions at $\sqrt{s}=8 \mathrm{TeV}$ with the ATLAS detector, arXiv: 1510.06218 [INSPIRE].

[90] Y. Bai and T.M.P. Tait, Searches with Mono-Leptons, Phys. Lett. B 723 (2013) 384 [arXiv: 1208.4361] [INSPIRE].

[91] N.F. Bell, J.B. Dent, A.J. Galea, T.D. Jacques, L.M. Krauss and T.J. Weiler, Searching for Dark Matter at the LHC with a Mono-Z, Phys. Rev. D 86 (2012) 096011 [arXiv:1209.0231] [INSPIRE].

[92] L.M. Carpenter, A. Nelson, C. Shimmin, T.M.P. Tait and D. Whiteson, Collider searches for dark matter in events with a $Z$ boson and missing energy, Phys. Rev. D 87 (2013) 074005 [arXiv: 1212.3352] [INSPIRE].

[93] A. Alves and K. Sinha, Searches for Dark Matter at the LHC: A Multivariate Analysis in the Mono-Z Channel, Phys. Rev. D 92 (2015) 115013 [arXiv:1507.08294] [INSPIRE].

[94] M. Neubert, J. Wang and C. Zhang, Higher-Order QCD Predictions for Dark Matter Production in Mono-Z Searches at the LHC, JHEP 02 (2016) 082 [arXiv:1509.05785] [INSPIRE].

[95] ATLAS collaboration, Search for dark matter in events with a $Z$ boson and missing transverse momentum in pp collisions at $\sqrt{s}=8 \mathrm{TeV}$ with the ATLAS detector, Phys. Rev. D 90 (2014) 012004 [arXiv: 1404.0051] [INSPIRE].

[96] Fermi-LAT collaboration, V. Vitale and A. Morselli, Indirect Search for Dark Matter from the center of the Milky Way with the Fermi-Large Area Telescope, arXiv:0912.3828 [INSPIRE].

[97] D. Hooper, C. Kelso and F.S. Queiroz, Stringent and Robust Constraints on the Dark Matter Annihilation Cross section From the Region of the Galactic Center, Astropart. Phys. 46 (2013) 55 [arXiv:1209.3015] [INSPIRE]. 